\title{
Maßnahmengesetzvorbereitungsgesetz und weitere Überlegungen zur Beschneidung des Umweltrechtsschutzes
}

\author{
Annette Guckelberger*
}

(C) Der/die Autor(en) 2020. Dieser Artikel ist eine Open-Access-Publikation.

Der Beitrag beleuchtet die Bestrebungen zur Beschneidung des Umweltrechtsschutzes durch das Maßnahmengesetzvorbereitungsgesetz sowie zu einer möglichen Rückkehr zur materiellen Präklusion vor dem Hintergrund des Unionsrechts und der AarhusKonvention.

\section{Einleitung}

Im Moment ist das Thema „Beschleunigung der Planungsund Genehmigungsverfahren“, das bereits in den 1990er Jahren eine bedeutsame Rolle spielte, ${ }^{1}$ wieder en vogue. ${ }^{2}$ Im März 2020 wurde das Maßnahmengesetzvorbereitungsgesetz $(\mathrm{MgvG})$ verkündet. ${ }^{3}$ Dadurch wird die Möglichkeit geschaffen, künftig über bestimmte Infrastrukturprojekte nicht mehr durch Planfeststellungsbeschluss, sondern durch Maßnahmengesetz zu entscheiden. Die in der Diskussion um das Gesetz zur weiteren Beschleunigung von Planungs- und Genehmigungsverfahren im Verkehrsbereich erhobenen Forderungen zur Wiedereinführung der materiellen Präklusion für alle Umweltrechtsbehelfe konnten sich (noch) nicht durchsetzen.

\section{Projektzulassungen durch Maßnahmengesetz}

Vermutlich weil man die Beschleunigung bei Verkehrsprojekten für so dringlich erachtete, wurde den zur Stellungnahme aufgeforderten Einrichtungen keine effektive Gelegenheit dafür (s. \$47 GGO) zum Referentenentwurf eines Genehmigungsbeschleunigungsgesetzes eingeräumt. Beispielhaft sei nur auf die Stellungnahme Nr. 47/2019 des Deutschen Anwaltvereins durch den Ausschuss Verwaltungsrecht verwiesen, wo es heißt: „Die Tatsache, dass ein Referentenentwurf mit einem so komplexe[n] Thema mit Bearbeitungsstand vom 16.10.2019 vorgelegt und gleichzeitig eine Stellungnahme bis zum 17.10.2019 erbeten wird, wird weder den Anforderungen an ein sorgfältiges und ordnungsgemäßes Gesetzgebungsverfahren noch der Weichenstellung gerecht, die objektiv mit dem Referentenentwurf verbunden ist. [...] Der Hinweis auf ein im Vorfeld eingeholtes juristisches Gutachten macht diese unterbliebene Erörterung nicht wett. “"

\subsection{Gesetzgebungsverfahren und Inhalt des $M g v G$}

Bereits am 8.11.2019 wurde sodann das Gesetzgebungsverfahren im Bundesrat des nunmehr unter anderer Bezeichnung firmierenden Entwurfs eines Gesetzes zur Vorbereitung der Schaffung von Baurecht durch Maßnahmengesetz im Verkehrsbereich (Maßnahmengesetzvorbereitungsge-

Prof. Dr. Annette Guckelberger

Inhaberin des Lehrstuhls für Öffentliches Recht,

Universität des Saarlandes,

Saarbrücken, Deutschland setz) eingeleitet. ${ }^{5}$ Hatten sich CDU/CSU und SPD in ihrem Koalitionsvertrag für die 19. Legislaturperiode nur vorgenommen, bei fünf Pilotprojekten Baurecht durch Maßnahmengesetz zu erproben, ${ }^{6}$ werden in dem letztlich verabschiedeten Gesetz u.a. wegen des Klimaschutzprogramms 2030 der Bundesregierung zur Ermöglichung der Genehmigung von Schienenverkehrsprojekten durch Gesetz $z^{7}$ nunmehr 13 (s. die Nr. 6a) in den Anwendungsbereich des Gesetzes fallende Verkehrsinfrastrukturprojekte aufgezählt (\$2 S. $1 \mathrm{MgvG})$. Gemäß $\$ 1 \mathrm{MgvG}$ schafft dieses Gesetz ein Verfahren, um anschließend den Neu- oder Ausbau sowie die Änderung von Verkehrsinfrastruktur durch Gesetz anstatt durch Verwaltungsakt zuzulassen. Mithin handelt es sich bei dem MgvG um ein Vorschaltgesetz, um später über näher bestimmte Vorhaben durch Maßnahmengesetz entscheiden zu können. ${ }^{8}$ Ziel dieser Neuerung soll es sein, die Akzeptanz der Bevölkerung für die in diesem Gesetz bezeichneten Vorhaben zu steigern sowie ihre Realisierung zu beschleunigen. ${ }^{9}$

Die abschließende Aufzählung bezweckt die Sicherstellung, dass keine originären Verwaltungsfunktionen auf die Legislative übertragen werden, sondern gesetzliche Genehmigungsentscheidungen nur bei einzelnen, besonders ausgewählten Projekten erfolgen. ${ }^{10}$ Der Ausnahmecharakter wird mit der beschleunigten Schaffung der Infrastrukturprojekte, die aus Gründen des Klimaschutzes notwendig seien, und deren Einstufung als vordringlicher Bedarf mit zusätzlicher Ausweisung einer Engpassbeseiti-

*) Es handelt sich um den letzten von fünf Beiträgen, welche einer Langfassung ihres Vortrag in Berlin am 2.12.2019 auf dem „Forum Umweltrechtsschutz 2019: Erfahrungen mit der Novelle des Umwelt-Rechtsbehelfsgesetzes (UmwRG) seit 2017“, veranstaltet durch das Unabhängige Institut für Umweltfragen, entsprechen.

1) S. nur Ziekow (Hrsg.), Beschleunigung von Planungs- und Genehmigungsverfahren, 1998.

2) S. etwa auch das Gesetz zur Beschleunigung des Energieleitungsausbaus v. 13.5.2019, BGBl. I S. 706 ff. und dazu Baumann/Bri gola, DVB1. 2020, $324 \mathrm{ff.;}$ Schlacke/Römling, DVB1. 2019, $1429 \mathrm{ff}$. Ferner zu aktuellen Beschleunigungsvorschriften Stüer, DVBl. 2020, $617 \mathrm{ff}$.

3) Gesetz zur Vorbereitung der Schaffung von Baurecht durch Maßnahmengesetz im Verkehrsbereich (Maßnahmengesetzvorbereitungsgesetz - MgvG) v. 22.3.2020, BGBl. I S. $640 \mathrm{ff}$.

4) Stellungnahme Nr. 47/2019 des Deutschen Anwaltvereins durch den Ausschuss Verwaltungsrecht, Stand 8.1.2020, abrufbar unter https://anwaltverein.de/de/newsroom/sn-47-19-genehmigungsbeschleunigungsgesetz.

5) BR-Drs. 579/19.

6) Koalitionsvertrag 19. Legislaturperiode, S. 75 Z. 3426.

7) Klimaschutzprogramm 2030 der Bundesregierung zur Umsetzung des Klimaschutzplans 2050, S. 64.

8) Reidt, EurUP 2020, 86, der zutreffend hervorhebt, dass das MgvG selbst kein Maßnahmengesetz ist.

9) BR-Drs. 579/19, S. 8

10) BR-Drs. 579/19, S. 12. 
gung erläutert. ${ }^{11}$ In einer Stellungnahme im Rahmen der Anhörung des Ausschusses für Verkehr und digitale Infrastruktur wurde betont, dass es auf keinen Fall zu einer grundsätzlichen Aufgabenverlagerung von der Exekutive auf die Legislative kommen, sondern eine solche Verfahrensweise nur bei eingehend begründeten Ausnahmefällen möglich sein solle. Wenn daher der Pilot- oder Modellcharakter der erfassten Vorhaben betont werde, gehe es vor allem um die Sammlung von Erfahrungen mit vom Deutschen Bundestag getroffenen Zulassungsentscheidungen, insbesondere im Hinblick auf deren größere Akzeptanz und die damit einhergehende Beschleunigungswirkung. ${ }^{12}$ Ohne tatsächlich derartige Erfahrungen zu sammeln, hat man sich aber im zwischenzeitlich beschlossenen Strukturstärkungsgesetz Kohleregionen in Artikel 4 zur Aufnahme eines $\$ 2 \mathrm{a} \mathrm{MgvG}$ entschlossen, in welchem 16 weitere Verkehrsinfrastrukturprojekte genannt werden, die der Deutsche Bundestag mit dem Ziel der Strukturförderung der ehemaligen Kohleregionen durch Maßnahmengesetz anstelle eines Verwaltungsakts zulassen können soll. ${ }^{13}$

In Abweichung $\mathrm{zu}$ dem nicht zwingend ausgestalteten $\$ 25$ Abs. 3 VwVfG besteht nach $\$ 5 \mathrm{MgvG}$ eine Pflicht zur Durchführung einer frühen Öffentlichkeitsbeteiligung, die vor Einleitung des vorbereitenden Verfahrens stattzufinden hat. ${ }^{14}$ Die Zulassung der Vorhaben durch Maßnahmengesetz kommt nur nach vorheriger Durchführung eines vorbereitenden Verfahrens in Betracht. In dessen Rahmen hat eine Unterrichtung über den Untersuchungsrahmen nach $\$ 6 \mathrm{MgvG} \mathrm{zu}$ erfolgen, wobei wiederum in Abweichung zum regulären Planfeststellungsverfahren die zuständige Behörde gemäß $₫ 6$ Abs. $5 \mathrm{MgvG}$ der betroffenen Öffentlichkeit zwingend Gelegenheit zur Teilnahme am sog. Scoping-Termin zu geben hat, um Gegenstand, Umfang und Methoden der Umweltverträglichkeitsprüfung zu besprechen. ${ }^{15}$ Es folgt das in $\$ 7 \mathrm{MgvG}$ geregelte Anhörungsverfahren mit zwingender Durchführung eines Erörterungstermins. ${ }^{16}$ Hinsichtlich des Prüfungsumfangs kommt es im vorbereitenden Verfahren zu keinen Abweichungen gegenüber den herkömmlichen Verfahren, insbesondere keiner Absenkung der Umweltstandards. ${ }^{17}$ Auf diese Weise sollen die Verpflichtungen aus den Bestimmungen zur Öffentlichkeitsbeteiligung nach Art. 2 Abs. 5 Richtlinie 2014/52/EU umgesetzt werden. ${ }^{18}$ Die Verfahrensausgestaltung wurde zur Vermeidung materiell-rechtlicher und verfahrenstechnischer Defizite im Hinblick auf die Umweltverträglichkeitsprüfung und den Gebiets- und Artenschutz eng an das Planfeststellungsverfahren angelehnt. ${ }^{19}$

Bestehen nach Einschätzung der zuständigen Behörde nach Abschluss des Anhörungsverfahrens keine triftigen Gründe für die Annahme, dass die Zulassung des Verkehrsinfrastrukturprojekts durch ein Maßnahmengesetz erfolgen sollte, leitet sie dem Bundesministerium für Verkehr und digitale Infrastruktur einen begründeten Entscheidungsvorschlag zu, damit dieses über die Veranlassung eines Gesetzgebungsverfahrens für ein Maßnahmengesetz befinden kann ( $\$ 7$ Abs. $2 \mathrm{MgvG}$ ). Wie man an Satz 2 erkennen kann, wonach das Ministerium abweichend von dem Vorschlag der zuständigen Behörde von der Durchführung eines Gesetzgebungsverfahrens für ein Maßnahmengesetz absehen kann, wenn durch dieses die Zulassung des Verkehrsinfrastrukturprojekts zugunsten des Gemeinwohls nicht oder nur unwesentlich beschleunigt wird, bildet die zu erwartende nicht unwesentliche Beschleunigung den Maßstab für den Entscheidungsvorschlag und die Entscheidung des Ministeriums. ${ }^{20}$ Abzuwarten bleibt, inwieweit die Behörden sich gegen eine solche Überführung des Verfahrens in die Maßnahmengesetzgebung aussprechen werden, da in der Aufnahme der Vorhaben in das MgvG die Einschätzung und auch der Wille des Gesetzgebers zum Ausdruck kommt, möglichst durch Gesetz über diese zu entscheiden.
Soll die Zulassung des Vorhabens durch Maßnahmengesetz erfolgen, erstellt die zuständige Behörde einen Abschlussbericht ( $\$ 8 \mathrm{MgvG})$. Dieser enthält in der Regel die Planunterlagen mit allen entscheidungserheblichen Unterlagen ( $\$ 8$ Abs. 1 S. $2 \mathrm{MgVG}$ ). Er soll in seinem Aufbau und Inhalt einem Planfeststellungsbeschluss des jeweiligen Projekts entsprechen und mindestens die in $\$ 8$ Abs. $3 \mathrm{~S} .2$ MgvG aufgezählten Angaben umfassen. Nach Satz 3 muss der Bericht so konzipiert werden, dass durch ihn die Entscheidung des Bundestages nicht vorweggenommen wird, und gemäß Satz 4 muss er soweit wie möglich Raum für eine eigene Abwägung des Gesetzgebers lassen. Auf dieser Basis hat der Bundestag eine eigenständige Abwägungsentscheidung zu treffen. ${ }^{21}$ Während des Gesetzgebungsverfahrens befürwortete u.a. die FDP-Fraktion die Involvierung des Gesetzgebers bei der Planung großer Verkehrsprojekte, da dabei ,auch immer Fragen mit politischen Dimensionen zu entscheiden seien". ${ }^{22}$ In einer Stellungnahme im Rahmen der Anhörung des Verkehrsausschusses hieß es: „Da das Plenum die komplexe Abwägung unterschiedlichster Belange nicht wird leisten können, wird diese Aufgabe - jedenfalls in der derzeitigen Organisation des Deutschen Bundestages - auf den Verkehrsausschuss zukommen“. ${ }^{23}$ Aus der Zeitperspektive sei es sinnvoll, dass sich die Abgeordneten der federführenden Ausschüsse nicht erst im üblichen Gang des parlamentarischen Verfahrens mit dermaßen komplexen Fragen befassen, sondern sie am besten selbst bereits an Terminen der Öffentlichkeitsbeteiligung teilnehmen würden. ${ }^{24}$ $\mathrm{Ob}$ dies die Abgeordneten der federführenden Ausschüsse angesichts ihrer vielfältigen Aufgaben tatsächlich zu leisten vermögen, dürfte jedoch zweifelhaft sein, zumal ja noch die Entscheidung, ob über das Vorhaben durch Maßnahmengesetz entschieden werden soll, aussteht. Würde dies verneint, wäre ihre Teilnahme an der Öffentlichkeitsbeteiligung unnötig gewesen. Berücksichtigt man, dass sich die Zahl der möglichen Vorhaben, die für eine Entscheidung durch ein Maßnahmengesetz in Betracht kommen, durch das Strukturstärkungsgesetz Kohleregionen deutlich erhöht wurde, gibt es gute Gründe für die Annahme einer Überforderung des parlamentarischen Gesetzgebungsverfahrens. ${ }^{25}$

11) BR-Drs. 579/19, S. 13

12) Stellungnahme von Ziekow für den Deutschen Bundestag, Ausschuss für Verkehr und digitale Infrastruktur, Ausschussdrucks. 19(15)308-A.

13) Strukturstärkungsgesetz Kohleregionen v. 8.8.2020, BGB1. I S. 1795 ff.; s. auch BT-Drs. 19/20714 (neu), S. 18

14) BT-Drs. 19/16907, S. 23; Groß, JZ 2020, 76, 77; Ziekow, NVwZ 2020, 677, 679 .

15) BT-Drs. 19/16907, S. 23; Ziekow, NVwZ 2020, 677, 679

16) BT-Drs. 19/16907, S. 23

17) BR-Drs. 579/19, S. 18

18) BR-Drs. 579/19, S. 17

19) BR-Drs. 579/19, S. 18; s. auch Reidt, EurUP 2020, 86, 88

20) S. auch Ziekow, NVwZ 2020, 677, 679.

21) Stellungnahme von Ziekow für den Deutschen Bundestag, Ausschuss für Verkehr und digitale Infrastruktur, Ausschussdrucks. 19(15)308-A; kritisch gegenüber dieser Ausgestaltung, weil dadurch das Maßnahmengesetz des Parlaments vom „Gnaden der Exekutive abhängt", Stellungnahme von Posch für den Deutschen Bundestag, Ausschuss für Verkehr und digitale Infrastruktur, Ausschussdrucks. 19(15)308-B. Anders Stellungnahme der IHK Nord für den Deutschen Bundestag, Ausschuss für Verkehr und digitale Infrastruktur, Ausschussdrucks. 19(15)308-C, weil dadurch flexibel auf Änderungen der Sach- und Rechtslage reagiert werden könne.

22) Wiedergabe laut BT-Drs. 19/16907, S. 21; s. auch BTDrs. 19/17093, S. 3 unter Nr. 5.

23) Stellungnahme von Ziekow für den Deutschen Bundestag, Ausschuss für Verkehr und digitale Infrastruktur, Ausschussdrucks. 19(15)308-A.

24) Ziekow, NVwZ 2020, 677, 680.

25) So bereits für das StandAG Gärditz, in: FS für Wilfried Erbguth, 2019, 479, 498f. 
Auch ist die Frage aufzuwerfen, ob ein solches Verhalten bei der Beurteilung über die nicht unwesentliche Beschleunigung einer Entscheidung per Maßnahmengesetz Bedeutung erlangen könnte. Dafür bedürfte es aufjeden Fall der Dokumentation und dürfte allein die Teilnahme eines oder weniger Mitglieder des Ausschusses kaum ausreichen.

Möglicherweise hat sich der Gesetzgeber durch die Rechtslage in Dänemark inspirieren lassen, weil dort Großvorhaben durch Baugesetz planungsrechtlich zugelassen werden. ${ }^{26}$ Dass aber rechtsvergleichende Anleihen in der Begründung des Gesetzentwurfs fehlen, lässt sich damit erklären, dass sich die Rechtslage und die Verhältnisse in Deutschland zu stark von denen in Dänemark unterscheiden. ${ }^{27}$ Im Zuge seiner rechtsvergleichenden Betrachtung äußerte Siegert Zweifel, ob die Zulassung von Vorhaben durch ein Maßnahmengesetz zu einer schnelleren planungsrechtlichen Zulassung als ein Planfeststellungsbeschluss führen wird. ${ }^{28}$ Eine Beschleunigung lässt sich seiner Ansicht nach nur durch ein Zurückfahren des Detaillierungsgrads der Zulassungsentscheidungen erreichen. ${ }^{29}$

Leider findet man in der Begründung des Gesetzentwurfs keine eingehendere Erklärung, woraus sich die beschleunigte Realisierung der Vorhaben ergibt, zumal man auf die zwingende Ausgestaltung der Verfahrensschritte im vorbereitenden Verfahren Wert legte. Da zunächst die zuständige Behörde eine begründete Entscheidung über die Eignung des Projektes für eine Zulassungsentscheidung durch Maßnahmengesetz zu treffen und dann nochmals das zuständige Bundesministerium eigenständig hierüber $\mathrm{zu}$ befinden hat, bevor es zur Einleitung des Gesetzgebungsverfahrens kommt, handelt es sich hierbei um weitere - gegenüber den herkömmlichen Zulassungsverfahren - verzögernde Verfahrensschritte. ${ }^{30}$ Soll durch Maßnahmengesetz entschieden werden, muss die zuständige Behörde nach $\ 8$ MgvG zunächst einen an Aufbau und Inhalt eines Planfeststellungsbeschlusses orientierten Abschlussbericht erstellen, während sie im gewöhnlichen Verfahren ihre Planfeststellungsentscheidung treffen und bekanntmachen würde. ${ }^{31}$ Aus den Empfehlungen der Ausschüsse des Bundesrates ergibt sich, dass wohl von Seiten der Gesetzesverfasser von einem Beschleunigungseffekt durch eine Verkürzung des Rechtsschutzes ausgegangen wird. ${ }^{32}$ Ausgangspunkt dürfte sein, dass bei einer Zulassung der Vorhaben durch Gesetz anstelle einer solchen durch Verwaltungsakt der Verwaltungsrechtsweg ausgeschlossen ist. ${ }^{33}$ Die bislang überwiegende Rechtsprechung geht davon aus, dass der Gesetzgeber nicht unter den Begriff der öffentlichen Gewalt i.S.d. Art. 19 Abs. 4 S. 1 GG fällt. ${ }^{34}$ Den von den Maßnahmengesetzen betroffenen Individuen würde somit als Rechtsschutzmöglichkeit nur noch der Gang nach Karlsruhe vor das BVerfG verbleiben, also die Einlegung eines außerordentlichen Rechtsbehelfs in Form der Verfassungsbeschwerde, bei dem eine Überprüfung der Maßnahme nur am Maßstab des Grundgesetzes möglich ist. ${ }^{35}$ Erklären lässt sich dies damit, dass nur das BVerfG, nicht aber ein Fachgericht, ein Parlamentsgesetz verwerfen darf. ${ }^{36}$

Auch wenn es in der Tat so ist, dass Zulassungsverfahren für Großvorhaben in Deutschland zu lange dauern und es daher gute Gründe für Überlegungen gibt, wie solche schneller durchgeführt werden können, ${ }^{37}$ verwundert es nicht, dass die Stellungnahmen zu dem Gesetzentwurf äußerst unterschiedlich ausfielen. So kritisierte die FDPFraktion die Beschränkung des Gesetzes auf einige wenige Projekte und befürwortete in einem Antrag dessen Ausweitung auf eine Reihe großer Bauprojekte von nationaler Bedeutung. ${ }^{38}$ In seiner auf den MgvG-Entwurf bezogenen Stellungnahme vom 20.12.2019 forderte der Bundesrat einerseits eine gewisse Erweiterung der in der Liste aufgezählten Vorhaben, ${ }^{39}$ sein Verkehrsausschuss insbesondere eine Einbeziehung der Energieinfrastruktur. ${ }^{40}$ Andererseits bat der Bundesrat gleich zu Beginn seiner Stellungnahme, im weiteren Gesetzgebungsverfahren die Vereinbarkeit des Gesetzentwurfs mit dem Unionsrecht und Art. 9 AK nochmals zu prüfen. ${ }^{41}$ Geht man davon aus, dass die sich aus dem Grundgesetz ergebende Rechtsschutzgarantie des Art. 19 Abs. 4 S. 1 GG altruistisch agierenden Umweltorganisationen nicht zugutekommt, bedeutete dies - so die Ausführungen in den Empfehlungen der Ausschüsse des Bundesrates - ,dass den anerkannten Umweltverbänden mit den Maßnahmengesetzen jegliche Möglichkeit zu einer gerichtlichen Überprüfung genommen wird, obwohl sie als gesellschaftliche Träger der Umweltaspekte de[m] Allgemeinwohl [...] dienen". ${ }^{42}$ Nach dem Gutachten, das dem Gesetzentwurf zugrunde liegt, sind Verbandsklagen bei Parlamentsgesetzen nicht notwendig, weil Art. 9 Abs. 2 AK sowie die damit im Kontext stehenden Unionsrichtli-

26) Siegert, UPR 2019, 468.

27) Zur fehlenden Konzentrationswirkung der Entscheidung des Gesetzgebers in Dänemark Stellungnahme von Posch für den Deutschen Bundestag, Ausschuss für Verkehr und digitale Infrastruktur, Ausschussdrucks. 19(15)308-B. Dazu, dass die Beschleunigung nicht aus der Zulassung durch Gesetz resultiert, sondern dem insgesamt schnelleren Verfahren in Dänemark, Reidt, EurUP 2020, 86, 92.

28) Siegert, UPR 2019, 468, 473

29) Siegert, UPR 2019, 468, 473

30) Dazu, dass das Gesetzgebungsverfahren nochmals Zeit in Anspruch nimmt, Stellungnahme von Zschiesche/Unabhängiges Institut für Umweltfragen für den Deutschen Bundestag, Ausschuss für Verkehr und digitale Infrastruktur, Ausschussdrucks. 19(15)308-D. S. auch Stellungnahme von Vittorelli/BUND für den Deutschen Bundestag, Ausschuss für Verkehr und digitale Infrastruktur, Ausschussdrucks. 19(15)308-G sowie BT-Drs. 19/16042, S. 2; Wegener, ZUR 2020, $195 \mathrm{f}$.

31) Reidt, EurUP 2020, 86, 88.

32) BR-Drs. 579/1/19, S. 2f.; s. auch Groß, JZ 2020, 76, 79; Wegener, ZUR 2020, 195. Dazu auch schon Lege, in: Merten/Papier (Hrsg.), Handbuch der Grundrechte Bd. III, 2009, \$66 Rdnr. 18; Stern, Das Staatsrecht der Bundesrepublik Deutschland, Bd. III/2, 1994, S. 734.

33) Vgl. auch Blümel, DVBl. 1997, 205, 207.

34) BVerfG, Urt. v. 25.6.1968 - 2 BvR 251/63, BVerfGE 24, 33 , 49 ff.; BVerfG, Urt. v. 18. 12.1968-1 BvR 638/64 u. a., BVerfGE 24, 367, 401 ff.; BVerfG, Beschl v. 8. 4.1987 - 2 BvR 909/82 u. a., NJW 1987, 3115, 3120; BVerfG, Beschl v. 30.6.2015 - 2 BvR 1282/11, NVwZ 2015, 1434, 1440; Enders, in: BeckOK GG, 41. Edition Stand: 15.5.2019, Art. 19 Rdnr. 59; Jarass, in: ders./ Pieroth, GG, 15. Aufl. 2018, Art. 19 Rdnr. 44; Ziekow, Vorhabenplanung durch Gesetz, 2020, S. $60 \mathrm{f}$, der auch zu Rechtsschutzanforderungen aus einzelnen Grundrechten Stellung nimmt; a. A. Schenke, in: Bonner Kommentar zum Grundgesetz, Bd. 6, 198. EL 2019, Art. 19 Abs. 4 Rdnr. 338 m.w. N.; s. auch Huber, in: von Mangoldt/Klein/Starck, GG, Bd. 1, 7. Aufl. 2018 , Art. 19 Abs. 4 Rdnr. 435.

35) Kment, in: FS für Jarass, 2015, S. 301, 308; s. auch Groß, JZ 2020, 76, $79 \mathrm{ff}$. einschließlich Ausführungen zur Überprüfung des MgvG im Wege der abstrakten Normenkontrolle; s. auch BRDrs. 579/1/19, S. 6; zum beschränkten Prüfungsmaßstab auch Firgau, Exekutivgesetze, 1996, S. $43 \mathrm{ff}$.

36) Ziekow, Vorhabenplanung durch Gesetz, 2020, S. 60; a. A. Wegener, ZUR 2020, 195, 201.

37) S. dazu auch $W_{y s k}$, in: Appel/Wagner-Kardenal, Verwaltung zwischen Gestaltung, Transparenz und Kontrolle, 2019, S. 27 ff., $38 \mathrm{f}$.

38) BT-Drs. 19/16040, S. 2; s. auch den Artikel von Schwenn, Schneller zu neuen Verkehrswegen, FAZ v. 10.1.2020, Wirtschaft, S. 15. Für einen generellen Paradigmenwechsel, wenn Entscheidungen mit Auswirkungen für Millionen von Menschen getroffen werden, Stellungnahme von Posch für den Deutschen Bundestag, Ausschuss für Verkehr und digitale Infrastruktur, Ausschussdrucks. 19(15)308-B.

39) BR-Drs. 579/19 (Beschluss), S. 3f. Für eine Einbeziehung von zwei weiteren Vorhaben Stellungnahme Posch für den Deutschen Bundestag, Ausschuss für Verkehr und digitale Infrastruktur, Ausschussdrucks. 19(15)308-C.

40) BR-Drs. 579/1/19, S. $9 \mathrm{f}$.

41) BT-Drs. 19/16405, S. 1.

42) BR-Drs. 579/1/19, S. 2; s. auch Wegener, ZUR 2020, 195. 
nien für den Gesetzgeber keine Geltung beanspruchen. ${ }^{43}$ Zwar sei in Art. 9 Abs. 2 AK, anders als bei dessen Absatz 3 nicht von Behörden die Rede, allerdings folge dies indirekt aus Art. 6 Abs. 2 lit. c) AK, wonach die Öffentlichkeit über die für die Entscheidung zuständige Behörde zu informieren ist. ${ }^{44}$

Lediglich was die Ermächtigung zum Erlass von Rechtsverordnungen für Änderungen des Maßnahmengesetzes anbetrifft, die dem Bundesministerium für Verkehr und digitale Infrastruktur erteilt wird $(\$ 11 \mathrm{MgvG})$, trifft $\$ 12$ MgvG eine Rechtsschutzregelung, dass über die Gültigkeit einer Rechtsverordnung nach $\int 11$ Abs. 1, 2 MgvG auf Antrag das BVerwG entscheidet und $\$ 47$ VwGO entsprechend anzuwenden ist. Erneut kommt es zu einer sonderprozessualen Regelung, weil die Normenkontrolle nach \$47 Abs. 1 VwGO den Oberverwaltungsgerichten obliegt und Rechtsverordnungen des Bundes nach dieser Vorschrift nicht normenkontrollfähig sind.

\subsection{Verfassungsrechtliche Bedenken}

Im Unterschied zu Dänemark, wo jeder gegen das Baugesetz unabhängig von einer Beteiligung am Anhörungsverfahren bei einem rechtlichen Interesse die gerichtliche Überprüfung des Vorhabens erreichen kann, ${ }^{45}$ wird sich erst noch erweisen müssen, ob die vom deutschen Gesetzgeber mit dem MgvG angedachte Verkürzung des Rechtsschutzes verfassungsgemäß sowie völker- und unionsrechtskonform ist. $\mathrm{Zu}$ Recht werden in den Empfehlungen der Ausschüsse des Bundesrates erhebliche Zweifel an der Verfassungsund Unionsrechtskonformität dieser Ausgestaltung angemeldet. Zwar hat das BVerfG in der Stendal-Entscheidung eine Planfeststellung durch Gesetz für mit dem Grundgesetz kompatibel gehalten. ${ }^{46}$ Hinsichtlich des Verhältnisses von Legislative und Exekutive argumentierte es, dass die Planung nicht als Vorgang der reinen Subsumtion eines bestimmten Lebenssachverhalts unter die Tatbestandsmerkmale einer generell-abstrakten Norm verstanden werden könne. ${ }^{47}$ Zwar sei üblicherweise der Verwaltung die Entscheidung über konkrete Fachplanungen zugewiesen, weil sie über den dafür erforderlichen Verwaltungsapparat und Sachverstand verfügt. ,[W] enn hierfür im Einzelfall [aber] gute Gründe bestehen, etwa weil die schnelle Verwirklichung des Vorhabens von besonderer Bedeutung für das Gemeinwohl ist", dürfe das Parlament im Wege eines Gesetzes eine solche Entscheidung an sich ziehen. ${ }^{48}$ Allerdings stammte das zu überprüfende Gesetz aus dem Jahre 1993, also einer Zeit, in der es zum einen die Staatszielbestimmung des Art. 20a GG zum Schutz der natürlichen Lebensgrundlagen und zum anderen die Aarhus-Konvention (AK) samt ihrer unionsrechtlichen Ausformungen noch nicht gab. ${ }^{49}$

In der Empfehlung der Ausschüsse des Bundesrates werden Zweifel angemeldet, ob hinsichtlich aller aufgezählten Verkehrsprojekte solche Ausnahmesituationen gegeben sind, da sich die heutige Lage von derjenigen unmittelbar nach der Wiedervereinigung unterscheidet. ${ }^{50}$ Richtigerweise handelt es sich bei der Zulassung einzelner, konkreter Infrastrukturvorhaben in Gesetzesform um Einzelfallgesetze. ${ }^{51}$ Lege vertritt in Bezug auf das Gesetz zur Südumfahrung Stendal, dass es dem Gesetzgeber dabei darum ging, ein Gesetz selbst zu vollziehen. ${ }^{52}$ Nach Art. 19 Abs. 1 S. 1 GG muss ein Gesetz allgemein sein und darf nicht nur für den Einzelfall gelten. Ausgehend von der Annahme, dass nach der BVerfG-Rechtsprechung Durchbrechungen hiervon möglich sind, wenn die Regelung des singulären Sachverhalts von sachlichen Gründen getragen wird, ${ }^{53}$ beruht das $\mathrm{MgvG}$ nach dem ihm zugrunde liegenden Gutachten auf der Annahme, dass zwar nicht das allgemeine Beschleunigungsziel des Verkehrsinfrastrukturausbaus, die Lage des Vorhabens im Raum oder seine überregionale Bedeutung zur Rechtfertigung derartiger Abweichungen aus- reicht. Anders sei die Lage jedoch bei hoch politisierten und zwischen politischen Akteuren umstrittenen Vorhaben zu beurteilen, die einer rein administrativen Behandlung zu „entwachsen“ drohen oder bei denen die Vorhaben aufgrund ihrer Systemrelevanz eine Entscheidung dermaßen wesentlicher Fragen durch den Gesetzgeber nahelegen. ${ }^{54}$ Bei genauer Betrachtung handelt es sich hier um äußerst vage Richtvorgaben, denn viele Infrastrukturvorhaben sind hochumstritten. $\mathrm{Ob}$ ein solches Kriterium einer verfassungsgerichtlichen Prüfung standhalten wird, muss sich erst noch erweisen. Stellt man darauf ab, dass der Wortlaut des Art. 19 Abs. 1 S. 1 GG keine Ausnahme vom Verbot des Einzelfallgesetzes vorsieht, liegt es jedenfalls nahe, erhöhte Anforderungen an derartige Abweichungen zu stellen. ${ }^{55}$ So kritisiert Lege die Stendal-Entscheidung, weil man die Konsequenzen der damit einhergehenden Rechtsschutzverkürzung sowie die Frage der Erforderlichkeit einer noch weiteren Zurücknahme des Rechtsschutzes zugunsten eines erhofften Planungszeitgewinns genauer hätte prüfen müssen, zumal der parlamentarische Betrieb für derartige Planungsentscheidungen gegenüber der Verwaltung weniger geeignet sei. ${ }^{56}$

In der Literatur aus früheren Zeiten wurde die Verfassungsmäßigkeit einer Planung durch den Gesetzgeber u.a. im Hinblick auf die Gewaltenteilung, den von Verfassungs wegen gebotenen effektiven Rechtsschutz bzw. das Rechtsstaatsgebot in Abrede gestellt. ${ }^{57}$ Als Argumente für eine Eröffnung von Rechtsschutz auch gegen den Gesetzgeber lässt sich anführen, dass der Verfassungsgeber Art. 19 Abs. 4 GG gerade nicht auf die Verwaltung beschränkt hat, und dessen Stellung am Ende des Grundrechtskatalogs sowie die Bindung aller drei staatlichen Gewalten an die Grundrechte

43) Ziekow, Vorhabenplanung durch Gesetz, 2020, S. 64

44) Ziekow, Vorhabenplanung durch Gesetz, 2020, S. 64

45) Siegert, UPR 2019, 468, 472; s. auch Stellungnahme von Vittorelli/BUND für den Deutschen Bundestag, Ausschuss für Verkehr und digitale Infrastruktur, Ausschussdrucks. 19(15)308-G.

46) BVerfG, Beschl. v. 17. 7.1996 - 2 BvF 2/93, BVerfGE 95, 1, 23 f

47) BVerfG, Beschl. v. 17. 7. 1996 - 2 BvF 2/93, BVerfGE 95, 1, 16.

48) BVerfG, Beschl. v. 17. 7.1996 - 2 BvF 2/93, BVerfGE 95, 1, 17; kritisch Durner, Konflikte räumlicher Planungen, 2005, S. 443, weil sich aus der Verfassung kein solches Regel-Ausnahme-Verhältnis herleiten lassen soll.

49) Zu Letzterem Wegener, ZUR 2020, 195, 200.

50) BR-Drs. 579/1/19, S. 3; s. auch Stellungnahme von Vittorelli/ BUND für den Deutschen Bundestag, Ausschuss für Verkehr und digitale Infrastruktur, Ausschussdrucks. 19(15)308-G.

51) Ziekow, Vorhabenplanung durch Gesetz, 2020, S. 22 f.; s. auch Stüer, DVB1. 2020, 617, 621.

52) Lege, in: Merten/Papier (Hrsg.), Handbuch der Grundrechte Bd. III, 2009, \66 Rdnr. 92.

53) BVerfG, Urt. v. 6.12.2016 - 1 BvR 2821/11 u.a., NJW 2017, 217, $241 \mathrm{f}$, Rdnr. 394, juris.

54) Ziekow, Vorhabenplanung durch Gesetz, 2020, S. 33 f. Weitere vage Kriterien bei ders, NVwZ 2020, 677, $678 \mathrm{f}$.

55) Lege, in: Merten/Papier (Hrsg.), Handbuch der Grundrechte Bd. III, 2009, \$66 Rdnr. 125. Nach Meessen, DÖV 1970, 314, 319 ist nur bei Akten von staatspolitischer Bedeutung eine Durchbrechung in Erwägung zu ziehen.

56) Lege, in: Merten/Papier (Hrsg.), Handbuch der Grundrechte Bd. III, 2009, §66 Rdnr. 93.

57) Wobei sich damals ein Beschleunigungseffekt auch aus dem Wegfall des förmlichen Verfahrens ergab. Für eine Verfassungswidrigkeit Ossenbühl, in: FS für Hoppe, 2000, S. 183, $192 \mathrm{ff}$. verlangt einen Rechtsschutzausgleich; Repkewitz, VerwArch 88, 1997, 137, 153 f.; Ronellenfitsch, DÖV 1991, 771, 779f. (der u.a. darauf verweist, dass auch planerische Entscheidungen teils gebundene Einzelentscheidungen enthalten); Stüer, DVB1. 1991, 1331, 1339ff.; am Vorliegen der triftigen Gründe bei den Investitionsmaßnahmengesetzen zweifelnd Blümel, DVB1. 1997, 205, 211; kritisch auch Stern, Das Staatsrecht der Bundesrepublik Deutschland, Bd. III/2, 1994, S. 734f. A. A. Durner, Konflikte räumlicher Planungen, 2005, S. $434 \mathrm{ff}$. 
(Art. 1 Abs. 3 GG) für eine weite Auslegung der Rechtsschutzgarantie streitet. ${ }^{58}$ Insbesondere wenn die Grenzen zwischen Akten der Exekutive und der Legislative verschwimmen und Einzelfallgesetze zunehmen, drängt sich eine Einbeziehung des Gesetzgebers in den Schutzbereich des Art. 19 Abs. 4 GG auf. Sonst hätte es - so Huber - ,,der Gesetzgeber in der Hand, durch die Wahl der Gesetzesform auch über die Möglichkeiten des Rechtsschutzes zu verfügen. Das ist von der Verfassung jedoch ersichtlich nicht gewollt." ${ }^{59}$ Auch wenn es im Regelfall richtig ist, dass nur das BVerfG wegen der Stellung des unmittelbar demokratisch legitimierten Gesetzgebers Parlamentsgesetze verwerfen darf, könnte in Konstellationen, in denen der Gesetzgeber eine der Verwaltung obliegende Entscheidung an sich zieht und er aufgrund des Unionsrechts vergleichbar der Verwaltung eine normativ angeleitete Entscheidung zu fällen hat, eine diesem Entscheidungsinhalt angepasste Gerichtskontrolle geboten sein. So sprechen sich mehrere Autoren im Schrifttum bei solchen Legalplanungen für eine Intensivierung der (verfassungs-) gerichtlichen Kontrolldichte aus. ${ }^{60}$

Gro $\beta$ ist jedenfalls der Ansicht, dass die in Anlehnung an die Stendal-Entscheidung ${ }^{61}$ vorgenommenen Aussagen zur Beschneidung des Rechtsschutzes zu kurz greifen, weil das BVerfG in späteren Entscheidungen die Rechtsschutzgarantie stärker akzentuiert habe. ${ }^{62}$ In einer Entscheidung zur Anerkennung der Zeugen Jehovas als Körperschaft des öffentlichen Rechts durch den bremischen Landesgesetzgeber habe das BVerfG entschieden, dass die Wahl eines Gesetzes anstelle einer Verwaltungsentscheidung zu einer erheblichen Einschränkung des gerichtlichen Rechtsschutzes führe, weil die Verfassungsgerichtsbarkeit keine tatsächlich wirksame gerichtliche Kontrolle bei (Einzelpersonen-)Gesetzen wegen des beschränkten Prüfungsmaßstabs auf die Verletzung spezifischen Verfassungsrechts vornehmen könne. ${ }^{63}$ Es sei nicht einzusehen, warum diese Aussagen zu einem Einzelpersonengesetz nicht auf Maßnahmengesetze zu übertragen sei, die viele Personen tangieren. ${ }^{64} \mathrm{Da}$ das BVerfG in der zuletzt genannten Entscheidung aber vor allem auf den Gewaltenteilungsgrundsatz und die gebundene Entscheidung des Gesetzgebers rekurrierte, tun sich Zweifel an der Aussagekräftigkeit dieser Entscheidung hinsichtlich der vorliegend zur Debatte stehenden Planungsvorhaben auf. Im Übrigen hebt Gro $\beta$ zutreffend hervor, dass sich die nach Art. 14 Abs. 3 GG zulässige Enteignung durch Gesetz auf einen überschaubaren Personenkreis bezieht, während einen Planfeststellungsbeschluss ersetzende Maßnahmengesetze Sachverhalte mit einer Vielzahl von Enteignungsbetroffenen und hoher Komplexität betreffen. ${ }^{65}$ Derartige vom Gesetzgeber zu treffende Entscheidungen werden heutzutage sehr stark vom Völker- und Unionsrecht angeleitet und geprägt. Deshalb soll hier zunächst geprüft werden, ob nicht bereits das Unionsrecht und die AK einen weitergehenden Rechtsschutz gebieten. ${ }^{66}$

\subsection{Unionsrechtliche Bedenken}

Jedenfalls vor der Änderung der UVP-Richtlinie schien die Zulassung von Projekten durch Maßnahmengesetze auf den ersten Blick unionsrechtskonform zu sein. Gemäß Art. 1 Abs. 4 UVP-Richtlinie gilt diese nicht für Projekte, die im Einzelnen durch einen besonderen einzelstaatlichen Gesetzgebungsakt genehmigt werden, da die mit dieser Richtlinie verfolgten Ziele einschließlich desjenigen der Bereitstellung von Informationen im Wege des Gesetzgebungsverfahrens erreicht werden. Nach Einschätzung der EU-Kommission unterfallen Legislativmaßnahmen angesichts der Umschreibung des Begriffs der Behörde nicht dem Aarhus-Übereinkommen. ${ }^{67}$ Einschränkend wies die Kommission jedoch auf die Rechtsprechung des EuGH in der Rechtssache Boxus u.a. hin. Danach verlören Art. 9 AK und Art. 11 UVPRichtlinie jegliche praktische Wirksamkeit, wenn der bloße Umstand der Genehmigung eines Projekts durch einen Ge- setzgebungsakt zum Ausschluss der Umweltrechtsbehelfe zur Überprüfung der materiell- und verfahrensrechtlichen Rechtmäßigkeit des Projekts führen würde. ${ }^{68}$ Voraussetzung für die Annahme eines solchen ist die Erteilung der Genehmigung durch einen besonderen Gesetzgebungsakt. Hinzukommen muss, dass der Gesetzgebungsakt wie eine Genehmigung alle für eine Umweltverträglichkeitsprüfung erheblichen, vom Gesetzgeber berücksichtigten Punkte des Projekts umfassen muss. Dies erklärt, warum das Gesetz Regelungen zum vorbereitenden Verfahren, einer frühen Öffentlichkeitsbeteiligung, der Unterrichtung über den Untersuchungsrahmen und ein Anhörungsverfahren enthält. ${ }^{69}$ Der nach Abschluss des Anhörungsverfahrens von der zuständigen Behörde erstellte Abschlussbericht samt Anlagen ist Grundlage für die Abwägungsentscheidung des Bundestages ( $\$ 8$ Abs. 3 S. 3, 4 MgvG).

Zwischenzeitlich wurde die Richtlinie 2011/92/EU jedoch durch die Richtlinie 2014/52/EU geändert. ${ }^{70}$ Dies hat zur Streichung des Art. 1 Abs. 4 UVP-Richtlinie 2011/92/EU geführt. Gemäß Art. 2 Abs. 5 UVP-Richtlinie 2014/52/EU können die Mitgliedstaaten unbeschadet des Art. 7, der Projekte mit grenzüberschreitendem Bezug betrifft, ein Projekt, das durch einen besonderen einzelstaatlichen Gesetzgebungsakt zugelassen wird, von den Bestimmungen dieser Richtlinie, die sich auf die Beteiligung der Öffentlichkeit beziehen, ausnehmen, jedoch nur unter der Voraussetzung, dass die Ziele dieser Richtlinie erreicht werden. Außerdem muss der Mitgliedstaat der Kommission alle zwei Jahre über die Fälle berichten, in denen von dieser Ausnahme Gebrauch gemacht wurde. Der EuGH entschied am 29.7.2019, dass die Genehmigung eines Projekts durch Gesetzgebungsakt ,die gleichen Merkmale wie eine [herkömmliche] Genehmigung [aufweisen muss]"“.71 Weil die Genehmigung hinreichend genau und abschließend ist,

58) Huber, in: von Mangoldt/Klein/Starck, GG, Bd. 1, 7. Aufl. 2018 Art. 19 Abs. 4 Rdnr. 435; Schulze-Fielitz, in: Dreier, GG, Band I, 3. Aufl. 2018, Art. 19 Abs. 4 Rdnr. 50; s. auch Schmidt-Aßmann, in: Maunz/Dürig, GG, 88. EL 08/2019, Art. 19 Abs. 4 Rdnr. 93. Groß, JZ 2020, 76, 80, wonach sonst Art. 19 Abs. 4 GG einem einfachen Gesetzesvorbehalt unterstellt würde. So aber vor allem hinsichtlich der Rechtsprechung Voßkuhle, Rechtsschutz gegen den Richter, 1996, S. $150 \mathrm{f}$.

59) Huber, in: von Mangoldt/Klein/Starck, GG, Bd. 1, 7. Aufl. 2018 , Art. 19 Abs. 4 Rdnr. 435; ebenso Ibler, in: Berliner Kommentar GG, III/2019, Art. 19 Abs. 4 Rdnr. 82; Schenke, in: Bonner Kommentar zum Grundgesetz, Bd. 6, 198. EL 2019, Art. 19 Abs. 4 Rdnr. 346. Ggf. für eine entsprechende Anwendung des Art. 19 Abs. 4 GG Kunig, Jura 1993, 308, 312.

60) Huber, in: von Mangoldt/Klein/Starck, GG, Bd. 1, 7. Aufl. 2018 , Art. 19 Abs. 4 Rdnr. 437; für eine intensivere Kontrolle des BVerfG, die aber nicht ganz so weit wie die verwaltungsgerichtliche Kontrolle reicht, Schmidt-Aßmann, in: Maunz/Dürig, GG, 88. EL 8/2019, Art. 19 Abs. 4 Rdnr. 95.

61) BVerfG, Beschl. v. 17. 7. 1996 - 2 BvF 2/93, BVerfGE 95, 1, 22.

62) Gro $\beta$, JZ 2020, 76, 79 f.; s. auch Wegener, ZUR 2020, 195, $196 \mathrm{ff}$.

63) BVerfG, Beschl. v. 30.6.2015 - 2 BvR 1282/11, BVerfGE 139, 321, 364; s. auch Kment, in: FS für Jarass, 2015, S. 308.

64) Gro $\beta$, Verfassungsblog v. 6.11.2019, Stand 13.11.2019, abrufbar unter https://verfassungsblog.de/rechtsschutz-gegen-verkehrsprojekte-als-stoerfaktor-einer-politik-der-maximalen-beschleunigung/.

65) $G r o \beta$, JZ 2020, 76, 79

66) Zu den mit dem Rechtsschutzausschluss verbundenen unionsund völkerrechtlichen Fragen auch BR-Drs. 579/1/19, S. 3.

67) Komm., Mitt. v. 28.4.2017, über den Zugang zu Gerichten in Umweltangelegenheiten, $\operatorname{COM}(2017)$ 2616, S. 48.

68) $\mathrm{EuGH}$, Urt. v. 18.10.2011 - C-128/09 bis C-131/09 u.a., Slg. 2011, I-9711, NuR 2011, 861, 863.

69) Dazu auch Ziekow, Vorhabenplanung durch Gesetz, 2020, S. $65 \mathrm{ff}$

70) Richtlinie 2014/52/EU des Europäischen Parlaments und des Rates vom 16.4.2014 zur Änderung der Richtlinie 2011/92/EU über die Umweltverträglichkeitsprüfung bei bestimmten öffentlichen und privaten Projekten, ABl. 2014 L 124, S. $1 \mathrm{ff}$

71) EuGH, Urt. v. 29.7.2019 - C-411/17, ECLI:EU:C:2019:622, Rdnr. 105, juris. 
muss der Gesetzgebungsakt wie eine gewöhnliche Genehmigung alle für die Umweltverträglichkeitsprüfung erheblichen, vom Gesetzgeber berücksichtigten Umstände umfassen. ${ }^{72}$ Des Weiteren muss der Gesetzgeber im Zeitpunkt der Genehmigung über ausreichende Angaben hinsichtlich des betreffenden Projekts verfügen. ${ }^{73}$ In Fortführung seiner Rechtsprechung in der Rechtssache Boxus judizierte der EuGH, dass das Vorliegen der Voraussetzungen dieser Norm gerichtlich überprüfbar sein muss, ,wobei sowohl der Inhalt des erlassenen Gesetzgebungsakts als auch das gesamte Gesetzgebungsverfahren, das zu seinem Erlass geführt hat, und insbesondere die Vorarbeiten und die parlamentarischen Debatten zu berücksichtigen sind“. ${ }^{74}$

Das vorbereitende Gutachten zum MgvG geht hinsichtlich der Vereinbarkeit mit dem Unionsrecht davon aus, dass Art. 2 Abs. 5 UVP-Richtlinie 2014/52/EU greife, wenn der Gesetzgeber ein konkretes Projekt von den Bestimmungen der UVP-Richtlinie über die Öffentlichkeitsbeteiligung ausnehme, dessen Zulassung durch einen besonderen Gesetzgebungsakt geschieht und die Entscheidung des Gesetzgebers zur eigenen Durchführung eines solchen Gesetzgebungsverfahrens erkennen lasse, wie in diesem Verfahren die Ziele der UVP-Richtlinie verwirklicht werden sollen. ${ }^{75}$ Diese Ausführung ist wohl so zu verstehen, dass das zuletzt genannte Kriterium bereits in der vorgelagerten Entscheidung erfüllt sein muss. Da Art. 11 UVPRichtlinie an die Regelungen über die Öffentlichkeitsbeteiligung anknüpft, erlange bei einem Vorgehen nach Art. 2 Abs. 5 UVP-Richtlinie 2014/52/EU diese Rechtsschutzbestimmung keine Bedeutung. ${ }^{76}$ Angesichts der EuGHRechtsprechung sei davon auszugehen, dass die Erfüllung der Voraussetzungen des Art. 2 Abs. 5 UVP-Richtlinie 2014/52/EU durch ein Gericht nachprüfbar sein müsse, wobei eine inzidente gerichtliche Kontrolle genüge. ${ }^{77}$ Leider wird dann aber in dem Gutachten nicht mehr dazu Stellung genommen, wie diese gerichtliche Kontrolle zustande kommen soll. ${ }^{78}$

Dies mag damit zusammenhängen, dass die vom EuGH bejahte Notwendigkeit gerichtlichen Rechtsschutzes kaum erläutert wird. Da Art. 11 UVP-Richtlinie die gerichtliche Überprüfung von Entscheidungen betrifft, für welche die Bestimmungen dieser Richtlinie über die Öffentlichkeitsbeteiligung gelten, geht es bei dieser Vorschrift gerade nicht um Bestimmungen i. S.d. Art. 2 Abs. 5 UVP-Richtlinie 2014/52/EU, die sich auf die Beteiligung der Öffentlichkeit beziehen (= diese wird in Art. 6 Abs. 4 UVP-RL ausgestaltet), sondern um eine davon zu unterscheidende Regelung zum Rechtsschutz. ${ }^{79}$ Ausgehend vom Sinn und Zweck des Art. 2 Abs. 5 UVP-Richtlinie 2014/52/EU, jedenfalls die Einhaltung der Ziele dieser Richtlinie auch bei Entscheidungen des Gesetzgebers kontrollierbar auszugestalten, sprechen gute Gründe dafür, derartige Entscheidungen ebenso der Rechtsschutzregelung des Art. 11 UVP-Richtlinie zu unterstellen. ${ }^{80}$ Auch die Kommission stellt sich in ihrem im November 2019 veröffentlichten Leitfaden zur Anwendung der Ausnahmen u. a. nach Art. 2 Abs. 5 UVP-Richtlinie 2014/52/EU auf den Standpunkt, es müsse sichergestellt sein, ,dass jeder derartige Gesetzgebungsakt in Übereinstimmung mit Artikel 11 der UVPRichtlinie gemäß nationalen Verfahrensvorschriften vor einem einzelstaatlichen Gericht [...] in Bezug auf seine materiellrechtliche und verfahrensrechtliche Rechtmäßigkeit anfechtbar sein muss". 81

Ferner sind Zweifel daran angebracht, dass Art. 2 Abs. 5 UVP-Richtlinie 2014/52/EU nur das Vorschaltgesetz, nicht aber die einzelnen Maßnahmengesetze betreffen soll. Die zitierten Entscheidungen des EuGH beziehen sich nicht auf vorgelagerte Entscheidungen, sondern auf das genehmigende Gesetz selbst. Dieses muss den Anforderungen der UVP-Richtlinie entsprechen und insoweit überprüfbar sein. Für diese Lesart lässt sich anführen, dass im Wortlaut des Art. 2 Abs. 5 UVP-Richtlinie 2014/52/EU im Singu- lar von einem Projekt die Rede ist, das durch einen besonderen einzelstaatlichen Gesetzgebungsakt zugelassen wird. Diese Ausgestaltung deutet darauf hin, dass vornehmlich dieser Akt auf die Erfüllung der Ziele des Art. 2 Abs. 5 UVP-Richtlinie 2014/52/EU hin zu überprüfen ist. Weil der Gesetzgeber anders als die Verwaltung nicht an das Gesetz gebunden, sondern gewohnt ist, sich von seinen eigenen Vorgaben jederzeit lösen zu können, ${ }^{82}$ legt die mit den unionsrechtlichen Regelungen intendierte Effektuierung des Umweltschutzes eher nahe, dass bei den konkreten gesetzlichen Genehmigungsentscheidungen der Projekte die Erfüllung der Ziele der Offentlichkeitsbeteiligung gewährleistet sein muss.

Zutreffend sprechen die Ausschüsse des Bundesrates die Empfehlung aus, dass ausgehend von der Judikatur des EuGH die Frage, ob der in Rede stehende Gesetzgebungsakt die Voraussetzungen des Art. 2 Abs. 5 UVP-Richtlinie 2014/52/EU erfüllt, von einem nationalen Gericht nach den nationalen Verfahrensvorschriften überprüfbar sein muss. ${ }^{83}$ Damit tut sich die Frage auf, wie diesen unionsrechtlichen Anforderungen entsprochen werden soll, zumal Umweltorganisationen nach der Intention des Gesetzes gerade vom Rechtsschutz ausgeschlossen sein sollen. Im Hinblick auf das beim BVerfG liegende Verwerfungsmonopol in Bezug auf Parlamentsgesetze verneinte Kment die Möglichkeit einer unionsrechtskonformen Auslegung des $\$ 40$ Abs. 1 VwGO. ${ }^{84}$ Die Urteilsverfassungsbeschwerde steht nach dem Wortlaut des Art. 93 Abs. 1 Nr. 4a GG nur den in ihren Grundrechten Verletzten offen und damit grundsätzlich nicht den Umweltschutzvereinigungen. ${ }^{85}$ Infolgedessen spricht sich Kment, allerdings in Bezug auf das Standortauswahlgesetz, für eine unmittelbare Anwendung des Art. 11 UVP-Richtlinie aus, befürwortet angesichts des Schutzes des Parlamentsgesetzgebers eine Rechtsschutzmöglichkeit für Umweltorganisationen beim BVerfG und fordert ein diesbezügliches Tätigwerden des Gesetzgebers. ${ }^{86}$

Zwar hat sich der Erste Senat des BVerfG in seinem Beschluss vom 6.11.2019 in Abkehr von der früheren Rechtsprechung im Falle einer Verdrängung der nationalen Grundrechte aufgrund einer vollständigen Determi-

72) EuGH, Urt. v. 29.7.2019 - C-411/17, ECLI:EU:C:2019:622, Rdnr. 106, juris.

73) EuGH, Urt. v. 29.7.2019 - C-411/17, ECLI:EU:C:2019:622, Rdnr. 109, juris.

74) EuGH, Urt. v. 29.7.2019 - C-411/17, ECLI:EU:C:2019:622, Rdnr. 110, juris, Kursivhervorhebung durch die Verfasserin.

75) Ziekow, Vorhabenplanung durch Gesetz, 2020, S. 68.

76) Ziekow, Vorhabenplanung durch Gesetz, 2020, S. $68 \mathrm{f}$.

77) Ziekow, Vorhabenplanung durch Gesetz, 2020, S. 69; ders., NVwZ 2020, 677, 682.

78) S. aber die späteren Ausführungen bei Ziekow, NVwZ 2020, 677, $684 \mathrm{f}$.

79) Ohne Begründung, aber Art. 11 UVP-RL für einschlägig erachtend Kment, in: FS für Jarass, 2015, S. 313; s. auch Wegener, ZUR 2020, 195, $202 \mathrm{ff}$.

80) S. auch Stellungnahme von Vittorelli/BUND für den Deutschen Bundestag, Ausschuss für Verkehr und digitale Infrastruktur, Ausschussdrucks. 19(15)308-G.

81) Bekanntmachung der Kommission vom 14.11.2019 Leitfaden zur Anwendung der Ausnahmen im Rahmen der Richtlinie über die Umweltverträglichkeitsprüfung (Richtlinie 2011/92/EU des Europäischen Parlaments und des Rates in ihrer durch die Richtlinie 2014/52/EU geänderten Fassung) - Art. 1 Abs. 3, Art. 2 Abs. 4 und 5, ABl. 2019 C 386, S. 12 unter Nr. 4.7.

82) Dazu auch Durner, Konflikte räumlicher Planungen, 2005, S. 448.

83) BR-Drs. 579/1/19, S. 5; s. auch Kment, in: FS für Jarass, 2015, S. 313.

84) Kment, in: FS für Jarass, 2015, S. $314 \mathrm{f}$.

85) Kment, in: FS für Jarass, 2015, S. $314 \mathrm{f}$.

86) Kment, in: FS für Jarass, 2015, S. 316f.; ein auf die Maßnahmengesetze bezogener Vorschlag einer solchen Regelung findet sich bei Stüer, DVB1. 2020, 617, 622 . 
nierung des nationalen Rechts durch das Unionsrecht für eine Überprüfung der Einhaltung der Unionsgrundrechte durch die deutschen Stellen einschließlich einer Anrufung des EuGH offen gezeigt. ${ }^{87}$ Allerdings betonte der Senat auch den Charakter der Verfassungsbeschwerde als auBerordentlichen Rechtsbehelf, der sich auf die Kontrolle der (Unions-) Grundrechte beschränke (s. auch den Wortlaut des Art. 93 Abs. 1 Nr. 4a GG). ${ }^{88}$ „Soweit also die Auslegung des Unionsrechts unabhängig von den Rechten der Charta in Frage steht, sind hierzu allein die Fachgerichte berufen und bleiben diese dementsprechend als letztentscheidende innerstaatliche Instanz gegebenenfalls auch vorlageverpflichtet." ${ }^{89}$ Hieran erkennt man klar und deutlich, dass sich das BVerfG gerade nicht als ein Gericht zur Prüfung der Einhaltung des Sekundärrechts durch die nationalen Stellen versteht..$^{90}$ An dieser Stelle sei nur an Art. 19 Abs. 1 UAbs. 2 EUV erinnert, wonach die Mitgliedstaaten die erforderlichen Rechtsbehelfe zur Gewährleistung eines wirksamen Rechtsschutzes in den vom Unionsrecht erfassten Bereichen schaffen müssen. Zutreffend meldet die Empfehlung der Ausschüsse des Bundesrates Zweifel daran an, ob ein ausschließlich auf Verfassungsrecht reduzierter gerichtlicher Prüfungsmaßstab noch als unionsrechtskonform angesehen werden kann. ${ }^{91}$ Die Ziele der UVP-Richtlinie werden nur i.S.d. Art. 2 Abs. 5 erreicht, wenn die Legislativentscheidung in materieller und verfahrensrechtlicher Hinsicht durch die Gerichte überprüfbar ist. Angesichts der rechtlichen Ausgestaltung der Verfassungsbeschwerde - Annahmeerfordernis, Geltendmachung einer Grundrechtsverletzung sowie eingeschränkter Prüfungsmaßstab - ist dies zu verneinen. ${ }^{92} \mathrm{Im}$ Übrigen wäre es sehr bedauerlich, wenn vornehmlich das Anliegen, die Rechtsschutzmöglichkeiten von Umweltvereinigungen gegen bestimmte Infrastrukturprojekte $\mathrm{zu}$ limitieren respektive eliminieren, nicht nur zu dem bereits aufgezeigten unübersichtlichen Sonderverwaltungsprozessrecht, sondern nunmehr auch zu einem Sonderverfassungsprozessrecht führen würde.

Es gibt deshalb gute Argumente für die Annahme, dass derartige Fragen durch die Verwaltungsgerichtsbarkeit überprüfbar sein müssen, zumal nach der EuGH-Rechtsprechung auch Umweltverbänden die in Art. 47 GRCh enthaltene Garantie auf einen wirksamen Rechtsbehelf zukommt. ${ }^{93}$ Da der Verwaltungsrechtsweg nach $\$ 40$ Abs. 1 VwGO zur Prüfung der Gültigkeit des beschlossenen Maßnahmengesetzes wegen Vorliegens einer verfassungsrechtlichen Streitigkeit nicht eröffnet ist, ${ }^{94}$ kommen als verwaltungsgerichtliche Rechtsschutzmöglichkeiten solche im Kontext der Realisierung des Inhalts der Maßnahmengesetze, insbesondere des Beginns der Bauarbeiten, und zwar sowohl im einstweiligen als auch im Hauptsacherechtsschutz in Betracht. ${ }^{95}$

In der Empfehlung der Ausschüsse für den Bundesrat wird als Hauptsacherechtsbehelf vor allem eine Feststellungsklage nach $\$ 43$ Abs. $1 \mathrm{VwGO}$ für möglich erachtet, ${ }^{96}$ wobei die Verwaltungsgerichte ggf. die Angelegenheit dem BVerfG nach Art. 100 Abs. 1 GG vorlegen ${ }^{97}$ oder bei Zweifeln an der Unionsrechtskonformität den EuGH gemäß Art. 267 AEUV einbeziehen können bzw. müssen. ${ }^{98}$ Insbesondere im Hinblick auf die Subsidiarität der Feststellungsklage gehen andere Überlegungen dahin, eine Klage auf Unterlassung des Vorhabens wegen Fehlens des erforderlichen Planfeststellungsbeschlusses, gegen die Entscheidung, dass ein Planfeststellungsbeschluss unterbleiben solle, oder auch auf Untersagung des formell illegalen Vorhabens $\mathrm{zu}$ erheben. ${ }^{99}$ Wegen Zweifeln hinsichtlich einer ausreichenden Ermächtigungsgrundlage für eine solche Untersagung ${ }^{100}$ halten andere auf jeden Fall die Beantragung eines gerichtlichen Baustopps für das durch Maßnahmengesetz zugelassene Projekt für möglich, wobei $\$ 75$ Abs. 2 S. 1 VwVfG aus unionsrechtlichen Gründen überspielt werde. ${ }^{101}$ Insoweit wird auf eine Entscheidung des BVerwG verwie- sen, nach welcher die Verbandsklagebefugnis gemäß dem UmwRG auch Rechtsbehelfe im Hinblick auf den Erlass behördlicher Aufsichtsmaßnahmen umfasst. Da das unmittelbare Klageziel der Umweltschutzvereinigung nicht im Erlass eines Verwaltungsakts, sondern in der Durchführung tatsächlicher Maßnahmen zur Verhinderung der Nutzung eines Radwegs bestand, hatte das BVerwG keine Zweifel am Rechtsschutzbedürfnis für eine solche Klage. ${ }^{102}$ Während aber das BVerwG in dem von ihm zu entscheidenden Fall wegen Absehens von einer Planfeststellung oder Plangenehmigung ohne weiteres die Eröffnung des Anwendungsbereichs des UmwRG wegen Unterlassens einer Entscheidung nach $\$ 1$ Abs. 1 S. 1 UmwRG bejahen konnte ( $\$ 1$ Abs. 1 S. 2 UmwRG), tut man sich bei Vorliegen eines Maßnahmengesetzes, das ja gerade eine allerdings nicht umweltrechtsbehelfsfähige Zulassungsentscheidung enthält, unter Zugrundelegung des Wortlauts des $\ 1$ Abs. 1 UmwRG und der Motivation des MgvG-Gesetzgebers, Umweltschutzvereinigungen bei diesen Vorhaben keinen Rechtsschutz zu eröffnen, auf den ersten Blick schwer. Auch $\$ 1$ Abs. 1 S. 1 Nr. 6 UmwRG, der sich auf Verwaltungsakte über Überwachungs- und Aufsichtsmaßnahmen „zur Umsetzung oder Durchführung von Entscheidungen nach Nr. 1-5“ bezieht, passt nach seinem Wortlaut bei einem Begehren auf behördliche Untersagung oder Stopp des gesamten Vorhabens nicht richtig, abgesehen davon, dass im Anwendungsbereich des Art. 9 Abs. 2 AK von der Beschränkung auf umweltbezogene Vorschriften abgesehen werden müsste. Diese Hürden lassen sich nur durch eine unionsrechtskonforme Auslegung des $\$ 1$ Abs. 1 S. 1, 2 UmwRG oder unter Rekurs auf den Anwendungsvorrang des Unionsrechts ausräumen. Da nach den bisherigen Erfahrungen mit dem UmwRG zwar einige, aber keinesfalls alle Verwaltungsgerichte zu diesbezüglichen Korrekturen des Enumerationsprinzips des $₫ 1$ Abs. 1 S. 1 UmwRG bereit sind, ${ }^{103}$ sind damit weitere Unwägbarkeiten für Rechtsschutz erstrebende Umweltvereinigungen verbunden.

87) BVerfG, Beschl. v. 6.11.2019 - 1 BvR 276/17, NJW 2020, 314, $318 \mathrm{Rdnr} .50 \mathrm{ff}$.

88) BVerfG, Beschl. v. 6.11.2019 - 1 BvR 276/17, NJW 2020, 314, 320 Rdnr. 73, 75.

89) BVerfG, Beschl. v. 6.11.2019 - 1 BvR 276/17, NJW 2020, 314 , 320 Rdnr. 75

90) S. auch Ziekow, NVwZ 2020, 677, 681.

91) BR-Drs. 579/1/19, S. 6; s. auch BR-Drs. 579/19(B), S. 2.

92) S. auch Stellungnahme von Vittorelli/BUND für den Deutschen Bundestag, Ausschuss für Verkehr und digitale Infrastruktur, Ausschussdrucks. 19(15)308-G sowie Wegener, ZUR 2020, 192, $199 \mathrm{f}$.

93) Z.B. EuGH, Urt. v. 20.12.2017 - Rs. C-664/15, ECLI:EU: C:2017:987, Rdnr. 45, 52; Guckelberger, Deutsches Verwaltungsprozessrecht unter unionsrechtlichem Anpassungsdruck, 2017, S. 138 f.; s. auch Groß, JZ 2020, 76, 82; Reidt, EurUP 2020, 86, 90 .

94) BR-Drs. 579/1/19, S. 6; Happ, in: Eyermann, VwGO, 15. Aufl. 2019, \43 Rdnr. 9b; s. auch Firgau, Exekutivgesetze, 1996, S. 43.

95) Reidt, EurUP 2020, 86, 90.

96) BR-Drs. 579/1/19, S. 7. S. auch Groß, JZ 2020, 76, 82. Stellungnahme von Ziekow für den Deutschen Bundestag, Ausschuss für Verkehr und digitale Infrastruktur, Ausschussdrucks. 19(15)308-A; ders., NVwZ 2020, 677, 684 weist aber darauf hin, dass eine solche Klage verwaltungsprozessrechtlich voraussetzungsreich ist.

97) BR-Drs. 579/1/19, S. 7; ebenfalls kritisch Fechter, jurisPRUmwR 12/2019, Anm. 1 unter G.

98) Dazu auch Reidt, EurUP 2020, 86, 90

99) Reidt, EurUP 2020, 86, 90; s. auch Ziekow, NvwZ 2020, 677, 684.

100) Näher dazu Ziekow, NVwZ 2020, 677, 684.

101) Ziekow, NVwZ 2020, 677, 684.

102) BVerwG, NVwZ 2017, 1634, $1635 \mathrm{f}$

103) Die Erfahrungen betreffen aber vor allem Art. 9 Abs. 3 AK, s. dazu Guckelberger, NuR 2020, 217, $224 \mathrm{f}$. 


\subsection{Konsequenzen des Unionsrechts für die verfassungsrechtliche Beurteilung}

Sollte sich die Lage derart präsentieren, stellt sich die Frage, ob das Maßnahmengesetzvorbereitungsgesetz ebenso wie die auf seiner Grundlage später ergehenden Maßnahmengesetze überhaupt ein geeignetes, erforderliches und angemessenes Mittel zur Erreichung der mit dem MgvG verfolgten Ziele ist bzw. sind. ${ }^{104}$ Geht man von einem verwaltungsgerichtlichen Inzidentrechtsschutz zur Überprüfung der Einhaltung des Art. 2 Abs. 5 UVP-Richtlinie 2014/52/EU aus, stellt sich insbesondere die Frage nach der Eingangsinstanz für einen derartigen Rechtsbehelf. Gemäß $\$ 50$ Abs. $1 \mathrm{Nr}$. $6 \mathrm{VwGO}$ ist das BVerwG für sämtliche Streitigkeiten, die Planfeststellungsverfahren und Plangenehmigungsverfahren für Vorhaben betreffen, die u.a. im AEG und BWStrG bezeichnet sind, erstinstanzlich zuständig. Das BVerwG interpretiert diese Vorschrift so, dass darunter alle Verfahren fallen, die einen unmittelbaren Bezug zu konkreten Planfeststellungs- oder Plangenehmigungsverfahren haben, die Streitigkeit mithin Teil der genehmigungsrechtlichen Bewältigung des Vorhabens ist. Darunter fasst es auch Streitigkeiten über Maßnahmen, die der späteren Planfeststellung zeitlich und sachlich vorausgehen (Vorbereitungsmaßnahmen) oder einen Ausschnitt der in einem laufenden Planfeststellungsverfahren zu lösenden Probleme darstellen, sowie Streitigkeiten, ob bestimmten Baumaßnahmen an dem betreffenden Verkehrsweg ein Planfeststellungs- oder Plangenehmigungsverfahren hätte vorausgehen müssen. ${ }^{105}$

Vorliegend würde es sich jedoch um Streitigkeiten handeln, die kein Planfeststellungsverfahren betreffen, sondern über deren Zulassung in anderer Weise, nämlich nach dem Verfahren des Maßnahmengesetzvorbereitungsgesetzes durch Maßnahmengesetz entschieden wurde. Auch wenn die Inzidentrechtsschutzverfahren letztlich darauf hinauslaufen, dass in deren Kontext die Rechtmäßigkeit der $\mathrm{Zu}-$ lassung des Vorhabens durch das Maßnahmengesetz geprüft und möglicherweise erreicht werden soll, dass über diese per Planfeststellungsbeschluss entschieden wird, fehlt diesen Streitigkeiten der unmittelbare Bezug zu einem Planfeststellungsverfahren. Deshalb würde $\int 50$ Abs. 1 Nr. 6 VwGO jedenfalls nach seinem Wortlaut nicht greifen. ${ }^{106}$ Dort ist gerade nicht ganz allgemein von Streitigkeiten hinsichtlich von im AEG oder BWStrG genannten Vorhaben die Rede. Auch aus verfassungsrechtlichen Gründen ist diese Ausnahmevorschrift eng zu interpretieren. ${ }^{107}$

Folge davon wäre oftmals die erstinstanzliche Zuständigkeit des Verwaltungsgerichts. ${ }^{108}$ Da nicht auszuschließen ist, dass später eine analoge Anwendung dieser Norm geltend gemacht wird, geht damit erneut gerichtlicher Klärungsbedarf einher, der Zeit in Anspruch nehmen wird. Im Interesse aller sind Zuständigkeitsvorschriften klar zu formulieren und hätte der Gesetzgeber, der jedenfalls während des Gesetzgebungsverfahrens auf die Rechtsschutzproblematik und die Möglichkeit einer Feststellungsklage aufmerksam gemacht worden sein dürfte, eine entsprechende Klarstellung der Rechtslage vornehmen können. Davon hat er aber keinen Gebrauch gemacht. Nach Einschätzung der Empfehlung der Ausschüsse des Bundesrates dürfte sich dadurch hinsichtlich der Verfahrenslaufzeit partiell sogar eine Verschlechterung gegenüber der bisherigen Rechtslage ergeben. ${ }^{109}$

Auf jeden Fall wäre zu empfehlen, zunächst die Verfassungs- und Unionsrechtskonformität erster, auf Basis des MgvG getroffener Vorhabenzulassungen durch Maßnahmengesetze aufgrund darauf bezogener Rechtsschutzbegehren klären zu lassen, ${ }^{110}$ anstelle vorschnell den Katalog für derartige Vorhaben zu erweitern, ohne zu wissen, ob solche Maßnahmengesetze einer gerichtlichen Prüfung standhalten. Dabei ist auch zu berücksichtigen, dass Vorschriften zur Unbeachtlichkeit von Fehlern bei Planfeststellungsbeschlüssen nach ihrem Wortlaut keine Anwendung auf Entschei- dungen durch Maßnahmengesetze finden. ${ }^{111}$ Die Rechtslage zum Inzidentrechtsschutz ist, wie hinsichtlich der Eröffnung des Anwendungsbereichs des UmwRG, aber auch in Bezug auf die Eingangsinstanz derartiger Rechtsschutzbegehren aufgezeigt wurde, nicht einfach zu erschließen. Insoweit könnten Nachjustierungen der einfachgesetzlichen Vorschriften zum Inzidentrechtsschutz sinnvoll sein, um zeitintensive Auseinandersetzungen zu vermeiden. Soweit die AK einschlägig ist (s. u.), verpflichtet Art. 3 Abs. 1 AK die Vertragsparteien zum Ergreifen der erforderlichen Gesetzgebungsmaßnahmen, um so einen klaren Rahmen für die Durchführung dieses Abkommens zu schaffen.

Auch wurde moniert, dass der Gesetzentwurf an den entscheidenden Stellen offen lässt und keine belastbaren Kriterien benennen kann, wie sich durch dieses Vorhaben wirksam den Herausforderungen des Klimawandels begegnen lässt. ${ }^{112}$ Zwar wurde in einzelnen Stellungnahmen innerhalb der Anhörung vor dem Verkehrsausschuss des Deutschen Bundestages begrüßt, dass endlich der demokratisch legitimierte Gesetzgeber die unmittelbare Verantwortung für derartige Vorhaben übernimmt. ${ }^{113}$ Wenn die tatsächliche Entscheidung aber von dem Verkehrsausschuss getroffen werden soll, weil das Plenum mit einer solch schwierigen Abwägung überfordert sei, und gerade im Hinblick darauf, dass die Tätigkeit der Abgeordneten normalerweise gestaltender und politischer Natur und nicht so stark an das Recht rückgebunden ist, vermag dieses Argument für sich allein ohne einen adäquaten Rechtsschutz nicht zu überzeugen. ${ }^{114}$ Zusammenfassend wird das mit dem MgvG verfolgte Anlie-

104) Zur Möglichkeit der Verzögerungen bei Schaffung eines Gesetzes und der Notwendigkeit der Gerichte, das Verfahren aufgrund Art. 100 Abs. 1 GG auszusetzen, Ibler, in: Berliner Kommentar GG, III/2019, Art. 19 Abs. 4 Rdnr. 87; s. auch Reidt, EurUP 2020, 86, 90.

105) BVerwG, NVwZ 2013, 1219 Rdnr. 8.

106) BR-Drs. $579 / 1 / 19$, S. 7.

107) VGH München, Beschl. v. 5.12.2016 - 8 A 16.40019, Rdnr. 9, juris.

108) Die Regelung in $\$ 7$ Abs. 4 S. 1 UmwRG, wonach das OVG zuständig ist, beschränkt sich Rechtsbehelfe gegen Entscheidungen nach $\$ 1$ Abs. 1 S. 1 Nr. 4 UmwRG oder deren Unterlassen.

109) BR-Drs. 579/1/19, S. 7; Stellungnahme von Zschiesche/Unabhängiges Institut für Umweltfragen für den Deutschen Bundestag, Ausschuss für Verkehr und digitale Infrastruktur, Ausschussdrucks. 19(15)308-D. S. zu den enttäuschten Erwartungen in Bezug auf den Zeitgewinn, den man sich von den Investitionsmaßnahmengesetzen erhofft hatte, Durner, Konflikte räumlicher Planungen, 2005, S. 438.

110) Zur Frage, ob es sich tatsächlich lohnt, bei einzelnen Vorhaben einen derartigen Weg zu beschreiten, Reidt, EurUP 2020, 86, 87, 92.

111) Reidt, EurUP 2020, 86, 89

112) BR-Drs. 579/1/19, S. 3; s. auch Stellungnahme von Posch für den Deutschen Bundestag, Ausschuss für Verkehr und digitale Infrastruktur, Ausschussdrucks. 19(15)308-B. Dazu, dass man über das Vorliegen der Voraussetzungen für ein Abweichen von dem Einzelfallgesetz kontrovers diskutieren kann, auch Reidt, EurUP 2020, 86, 92.

113) Stellungnahme von Funke/Pro Mobilität für den Deutschen Bundestag, Ausschuss für Verkehr und digitale Infrastruktur, Ausschussdrucks. 19(15)308-F; Stellungnahme von Sommer für den Deutschen Bundestag, Ausschuss für Verkehr und digitale Infrastruktur, Ausschussdrucks. 19(15)308-E.

114) Zweifelnd Reidt, EurUP 2020, 86, 89. Ähnlich Stellungnahme von Vittorelli/BUND für den Deutschen Bundestag, Ausschuss für Verkehr und digitale Infrastruktur, Ausschussdrucks. 19(15)308$\mathrm{G}$, wonach es fraglich sei, ob entsprechend den jeweiligen politischen Mehrheitsverhältnissen getroffene Entscheidungen mehr akzeptiert werden als von einer Behörde. Siehe auch Meessen, DÖV 1970, 314, 319, wonach die Gefahr von Pauschalentscheidungen und parteipolitisch motivierten Fehlentscheidungen besteht, wenn das Parlament selbst durch Gesetze Vollziehungsakte erlässt. Dazu, dass das Parlament für solche Entscheidungen strukturell weniger gut geeignet ist, schon Kunig, Jura 1993, 308, 313. 
gen, den verwaltungsgerichtlichen Rechtsschutz bei gewissen Infrastrukturprojekten partiell auszuschließen, kritisch betrachtet. Ist es wirklich sinnvoll, wenn es zwar keinen direkten Rechtsschutz gegen das jeweilige Maßnahmengesetz gibt, aber aus unionsrechtlichen Gründen jedenfalls ein Inzidentrechtsschutz zur Verfügung stehen muss, der zu einem späten Zeitpunkt einsetzt, voraussichtlich langwieriger wäre und dem angestrebten Ziel der Rechtsschutzverkürzung zuwiderläuft? Auch hinsichtlich des angestrebten Ziels, eine Steigerung der Akzeptanz in der Bevölkerung für diese Vorhaben aufgrund der Entscheidung durch das Parlament $\mathrm{zu}$ erreichen, ist das Gesetz als wenig dienlich einzustufen. ${ }^{115}$ Gerade die Rechtsschutzgarantie des Art. 19 Abs. 4 S. 1 GG belegt, dass den Gerichten bei der Beilegung von Konflikten eine zentrale Rolle zukommt. ${ }^{116}$ Eine - wenn auch nur durch eine Instanz erfolgende - intensive Überprüfung normativ angeleiteter Entscheidungen durch unabhängige Richter dient deren Akzeptanz. Da gerade im Bereich von Infrastrukturmaßnahmen das Beschleunigungspotenzial in Bezug auf Umweltrechtsbehelfe weitgehend ausgeschöpft ist, kann ein Beitrag zur Beschleunigung insbesondere durch eine adäquate personelle und sachliche Ausstattung der Verwaltungsgerichtsbarkeit erreicht werden. ${ }^{117}$ Schließlich ist es nicht gerade vertrauenserweckend, wenn Individualkläger und Umweltorganisationen zwar bei Kleinvorhaben einen effektiven gerichtlichen (Umwelt-)Rechtsschutz erlangen können, ihnen aber bei den viel bedeutsameren Großvorhaben gar kein fachgerichtlicher Rechtsschutz zur Verfügung stehen soll.

\subsection{Bedenken im Hinblick auf die $A K$}

Da sowohl der nationale als auch der Unionsgesetzgeber an die AK gebunden sind, dürfen die in Rede stehenden Vorschriften nicht im Widerspruch zu dieser ausgelegt werden. Zutreffend wird darauf aufmerksam gemacht, dass Art. 2 Nr. 2 S. 2 AK gar nicht den pauschalen Ausschluss bestimmter Staatsorgane von der Geltung der AK bezweckt, sondern darauf abzustellen ist, in welcher Eigenschaft die jeweilige Stelle tätig wurde. ${ }^{118}$ Die hier vertretene Haltung deckt sich mit einer Entscheidung des ACC vom 23.10.2013, bei der es sich auf den Standpunkt stellte, ,[i]n the present case, however, the Parliament is no longer, acting' in a legislative capacity, but rather as the , public autority" authorizing a project. [...] The Committee observes that if all large-scale projects were subject to parliamentary authorizations procedure and evoked article 2, paragraph 2, of the Convention, then there is a risk that important projects would never be subject to the public participation requirements of the Convention and this would run counter its objectives“. ${ }^{119}$ Wie man an dieser Aussage erkennen kann, kommt es für das Handeln in gesetzgebender Eigenschaft nicht auf die Bezeichnung der Maßnahme an, sondern ist dafür eine inhaltliche, materielle Betrachtung vorzunehmen. ${ }^{120}$

In derartigen Konstellationen müssen also u.a. Umweltschutzvereinigungen gemäß Art. 9 Abs. 2 S. 1 AK Rechtsschutz erlangen können. Der mit dem MgvG zunächst intendierte völlige Ausschluss des Rechtsschutzes von Umweltvereinigungen würde sich damit kaum in Einklang bringen lassen. Es wird sich erst erweisen müssen, inwieweit ein gegen Maßnahmengesetze eröffneter Inzidentrechtsschutz mit dieser Konventionsnorm in Einklang steht. Teilweise wird dies angenommen. ${ }^{121}$ In Bezug auf Art. 9 Abs. 3 AK meinte das ACC, dass dieser den Mitgliedstaaten zwar einen Spielraum bezüglich der Mitglieder der Öffentlichkeit, nicht aber gleichermaßen hinsichtlich der anfechtbaren Handlungen eröffne. Da die Vorschrift aber keine spezifischen Anforderungen dazu mache, in welchem Stadium eine Handlung angreifbar sein müsse, stellte es sich auf den Standpunkt, dass eine inzidente Überprüfung im Rahmen einer darauf basierenden Entscheidung ausreichen könne, aber nicht stets muss, etwa weil es keine nachgelagerten überprüfbaren Entscheidungen gibt. ${ }^{122}$ Es ist also keineswegs ersichtlich, dann aus der Möglichkeit des Inzidentrechtsschutzes auf die Vereinbarkeit mit Art. 9 AK $\mathrm{zu}$ schließen. Dafür, dass ein Inzidentrechtsschutz grundsätzlich konventionskonform sein könnte, spricht die offen gehaltene Formulierung in Art. 9 Abs. 2 AK ,Zugang zu einem Überprüfungsverfahren". Andererseits deutet der dort enthaltene Zusatz ,um die materiell-rechtliche und verfahrensrechtliche Rechtmäßigkeit von Entscheidungen [...] anfechten zu können" darauf hin, dass den Vertragsparteien der sich geradezu aufdrängende unmittelbare Rechtsschutz gegen die jeweilige Entscheidung vor Augen gestanden haben dürfte. Es ist nicht ausgeschlossen, dass das ACC die Rechtslage im Hinblick auf Art. 9 Abs. 2 AK anders als in Bezug zu Art. 9 Abs. 3 AK beurteilen wird. Denn Art. 9 Abs. 2 AK betont den weiten Zugang zu Gericht und enthält weiterhin die Regelung, dass dieser die Möglichkeit eines vorangehenden Überprüfungsverfahrens vor dem gerichtlichen Verfahren unberührt lässt. Auch diese Vorgabe bezieht sich direkt auf die Überprüfung der jeweiligen Entscheidung, darum geht es aber bei Forderungen nach einem Baustopp gerade nicht.

Im Ubrigen sei daran erinnert, dass Art. 9 Abs. 4 S. 1 AK einen angemessenen und effektiven Rechtsschutz vorgibt. Zwar lässt die Aarhus-Konvention ebenso wie die als deren Umsetzung anzusehende Vorgabe des Art. 11 Abs. 2 UVP-Richtlinie, wonach die Mitgliedstaaten festlegen, in welchem Verfahrensstadium die Entscheidungen angefochten werden können, hinsichtlich des Zeitpunkts des Rechtsschutzes einen Gestaltungsspielraum. Je später aber der Zeitpunkt des Rechtsschutzes hinausgeschoben wird, desto eher besteht die Gefahr der Schaffung irreversibler Maßnahmen und kann von einer effektiven Durchsetzung des Umweltrechts und von einem effektiven Rechtsschutz immer weniger die Rede sein, insbesondere wenn man auf den Zeitpunkt eines Baustopps abstellt. Auch besteht bei dem erst spät einsetzenden Zeitpunkt für den gerichtlichen Rechtsschutz die Gefahr, dass der sowohl von der AK als auch dem Unionsrecht angestrebte enge Bezug zwischen der Öffentlichkeitsbeteiligung und dem Rechtsschutz zu stark gelockert ist. ${ }^{123}$ Insgesamt lassen sich daher durchaus Argumente dafür finden, dass im Anwendungsbereich des Art. 9 Abs. 2 AK ein direkter Rechtsschutz gegen das Maßnahmengesetz eröffnet sein muss.

\subsection{Kommissionsvorschlag zur schnelleren Realisierung von TEN-V-Vorhaben}

Ohne Klärung der Rechtsschutzfrage ist ein unionsrechtskonformer Erlass der Maßnahmengesetze nicht möglich. Da im Moment auf Unionsebene über einen Vorschlag der EU-Kommission über die Straffung von Maßnahmen zur rascheren Verwirklichung des transeuropäischen Verkehrsnetzes beraten wird, ${ }^{124}$ wonach in Bezug auf die erfassten

115) BR-Drs. 579/1/19, S. 8; kritisch auch Groß, JZ 2020, 76, 82

116) Stellungnahme von Vittorelli/BUND für den Deutschen Bundestag, Ausschuss für Verkehr und digitale Infrastruktur, Ausschussdrucks. 19(15)308-G; s. zur Befriedungsfunktion auch BVerfG, Beschl. v. 14.1.2020 - 2 BvR 1333/17, Rdnr. 98, juris. 117) Rubel, Editorial JA 2/2020.

118) Epiney/Diezig/Pirker/Reitemeyer, Aarhus-Konvention, 2018, Art. 2 Rdnr. 10.

119) ACC/C/2011/61, Rdnr. $53 \mathrm{f}$.

120) Wegener, ZUR 2020, 195, $201 \mathrm{ff}$.

121) Keine Bedenken daran hat Ziekow, NVwZ 2020, 677, 683.

122) ACC, ZUR 2018, 23, 26.

123) S. etwa die Überlegungen zur Vermeidung einer Disproportionalität von Partizipation und Legalplanung beim Rechtsschutz nach dem StandAG Schlacke, in: Burgi, ATRS 2019, 347, 354.

124) $\operatorname{COM(2018)~} 277$ final; dazu auch Guckelberger, NWVB1. 2020, $133 \mathrm{ff}$. 
Vorhaben sowohl spezifische verfahrensrechtliche Vorgaben als auch solche zu den Behörden zu erwarten sind, wäre auch in dieser Hinsicht zu überlegen, ob der erwogene neue Weg nicht in Bälde zu weiteren unionsrechtlichen Problemen führen wird. Selbst wenn der Vorschlag der Kommission keine Rechtsschutzregelung enthält, findet sich bei den Gründen und Zielen des Vorschlags die Äußerung, dass die vorliegende Initiative im Hinblick auf die Durchführung von TEN-V-Infrastrukturvorhaben den Zugang zur Justiz gewährleistet. ${ }^{125}$ Der Vollständigkeit halber sei darauf hingewiesen, dass der EuGH die nationalen Gerichte hinsichtlich der Frage der Anwendung nationalen Umweltrechts bei der Durchführung eines Vorhabens von gemeinsamen Interesse aufgrund der Leitlinien für die transeuropäische Energieinfrastruktur für verpflichtet hielt, das nationale Verfahrensrecht so weit wie möglich im Einklang mit den Zielen der Art. 9 Abs. 3, 4 AK auszulegen (keine übermäßig teuren Gerichtsverfahren). ${ }^{126}$

\section{Wiedereinführung der materiellen Präklusion auch bei Art. 9 Abs. 2 AK in modifizierter Gestalt?}

Das Bundesministerium für Verkehr und Infrastruktur hat im Herbst 2019 zudem ein Rechtsgutachten zur Wiedereinführung der materiellen Präklusion im Anwendungsbereich von Art. 9 Abs. 2 AK erstellen lassen. ${ }^{127}$ Anders als erwartet war aber in dem am 8.11.2019 im Bundesrat eingebrachten Entwurf eines Gesetzes zur weiteren Beschleunigung von Planungs- und Genehmigungsverfahren im Verkehrsbereich ${ }^{128}$ keine Regelung zur Wiedereinführung der materiellen Präklusion enthalten. Erst im Zuge der Empfehlung der Ausschüsse des Bundesrates wurde der Wunsch geäußert, in das AEG, FStrG und das Bundeswasserstraßengesetz wieder eine Regelung zur materiellen Präklusion aufzunehmen, aber in modifizierter Gestalt gegenüber der vom EuGH 2015 beanstandeten Regelung. Nach diesem Vorschlag müssten Einwendungen in den Planfeststellungsverfahren bis zwei Monate nach Ablauf der Auslegungsfrist erhoben werden. Mit Ablauf der Einwendungsfrist wären sowohl für das Verfahren über die Zulässigkeit des Vorhabens als auch in einem nachfolgenden verwaltungsgerichtlichen Verfahren alle Einwendungen ausgeschlossen, die nicht auf besonderen privatrechtlichen Titeln beruhen und die auf Grundlage der ausgelegten Unterlagen vernünftigerweise hätten vorgetragen werden können. ${ }^{29}$ \$ 7 Abs. 4 UmwRG würde im Rechtsbehelfsverfahren gegen einen Planfeststellungsbeschluss oder eine Plangenehmigung keine Anwendung finden. ${ }^{130}$

Ziel der vorgeschlagenen Regelungen ist es, im Hinblick auf den Klimaschutz bestimmte Infrastrukturprojekte schneller voranzutreiben. ${ }^{131}$ Indem im Ergebnis ein Zeitraum von drei Monaten für die Erhebung von Einwendungen zur Verfügung steht, verspricht man sich eine Erhöhung der Qualität der Einwendungen und Stellungnahmen, ,so dass auf dieser Grundlage eine umfassendere Sachverhaltsaufklärung bei gesteigerter Prüftiefe und Problemlösung im Verwaltungsverfahren erfolgen kann“. ${ }^{132}$ Aus Sicht eines Informationsverwaltungsrechts lassen sich derartige Vorschriften als Informationsstoppregeln mit der Folge deuten, ${ }^{133}$ dass einer Zulassungsentscheidung ab einem bestimmten Zeitpunkt keine neuen Tatsachen oder Erkenntnisse mehr entgegengehalten werden können. ${ }^{134}$ Nach Meinung des empfehlenden Ausschusses sei durch diese Ausgestaltung sichergestellt, ,dass die Gerichte die Norm bezogen auf die Substantiierungslast im Einzelfall verhältnismäßig und damit unionsrechtskonform handhaben können“. 135

\subsection{Unionsrechtliche Zulässigkeit der Wiedereinführung der materiellen Präklusion?}

Zur Begründung dieser vor dem Hintergrund der UmwRG-Novelle überraschenden Wendung wird auf die Protect-Entscheidung des EuGH verwiesen, in der dieser die verfahrensrechtlichen Regelungen im Anwendungsbereich der AK unter Berücksichtigung der unionsrechtlichen Rechtsschutzgarantie weiter präzisiert hat. ${ }^{136}$ Der dortige Standpunkt des EuGH, dass Präklusionsregelungen dem von Art. 9 Abs. 4 AK vorgegebenen angemessenen, fairen, gerechten und zügigen Rechtsschutz Rechnung tragen würden und die Rechtsschutzgarantie des Art. 47 GRCh unter Wahrung des Verhältnismäßigkeitsgrundsatzes einschränkbar sei, treffe für andere, Art. 9 Abs. 2 AK unterfallende Präklusionsregelungen gleichermaßen zu. ${ }^{137} \mathrm{Da}$ nach Einschätzungen im Schrifttum die Rechtsprechung des EuGH tendenziell kasuistisch, kontextbezogen und regelmäßig auch nicht so ausführlich begründet sei, sei die Entwicklung divergierender Rechtsprechungslinien durch den EuGH für gewisse Übergangszeiträume nicht ungewöhnlich, bevor gewisse Grundsatzfragen im Zuge eines gerichtlichen Klärungsprozesses umfassend beantwortet würden. ${ }^{138}$ Mit anderen Worten geht man davon aus, dass die Rechtsprechung des EuGH zur materiellen Präklusion im Umweltrecht noch nicht ausreichend konsolidiert ist und hofft darauf, dass sich dieser in einem zweiten Anlauf von seiner Entscheidung in der Rechtssache C-137/14, in der er die materielle Präklusion in $\$ 2$ Abs. 3 UmwRG a.F. als unionsrechtswidrig einstufte, distanzieren wird.

Richtigerweise wird man den Ausgang eines solchen Verfahrens vor dem EuGH schwer einschätzen können, zumal die Ausgestaltungen von Art. 9 Abs. 2 und Abs. 3 AK variieren. So ist der Spielraum der Vertragsparteien aufgrund der in Art. 9 Abs. 3 AK im innerstaatlichen Recht festzulegenden Kriterien größer. Während Art. 9 Abs. 3 AK lediglich eine gerichtliche Kontrolle der Einhaltung umweltbezogener Rechtsvorschriften verlangt, ist die Uberprüfungsbefugnis der Gerichte bei Art. 9 Abs. 2 AK umfassend. Weil Art. 11 Abs. 3 UVP-Richtlinie und Art. 9 Abs. 2 UAbs. 2 AK die Notwendigkeit eines weiten Gerichtszugangs betonen und dadurch der Ermessensspielraum der Mitgliedstaaten eingeschränkt wird, ${ }^{139}$ eine solche Formulierung aber bei Art. 9 Abs. 3 AK fehlt, gibt es durchaus eine Reihe von Argumenten zur plausiblen Erklärung der unterschiedlichen Bewertung der Unionsrechtskonformität der materiellen Präklusion durch den EuGH. ${ }^{140}$ Angesichts dessen,

125) $\operatorname{COM}(2018) 277$ final, S. 3.

126) EuGH, Urt. v. 15.3.2018 - C-470/16, ECLI:EU:C:2018:185, Rdnr. 57, juris.

127) $\operatorname{COM}(2018) 277$ final, S. 3.

128) BR-Drs. 582/19.

129) BR-Drs. 582/1/19 (Ausschussempfehlung), S. 3 ff.

130) BR-Drs. $582 / 1 / 19$, S. 4 ff.

131) BR-Drs. 582/1/19, S. 6.

132) BR-Drs. $582 / 1 / 19$, S. 7.

133) Durner, Rechtsgutachten zur Wiedereinführung der materiellen Präklusion v. 7.10.2019, S. 41

134) Durner, Rechtsgutachten zur Wiedereinführung der materiellen Präklusion v. 7.10.2019, S. 42.

135) BR-Drs. 582/1/19, S. 7.

136) BR-Drs. $582 / 1 / 19$, S. 8.

137) S. auch Durner, Rechtsgutachten zur Wiedereinführung der materiellen Präklusion v. 7.10.2019, S. 48 ff.; s. auch, aber ohne Bezug auf den Gesetzentwurf, Deutsch, DVB1. 2019, 1437, 1443

138) Durner, Rechtsgutachten zur Wiedereinführung der materiellen Präklusion v. 7.10.2019, S. 52; allgemein zur „Pendelrechtsprechung" des EuGH Kment, in: ders. (Hrsg.), Der Einfluss des Europäischen Gerichtshofs auf das Umwelt- und Infrastrukturrecht, 2020, 1, 14f.

139) Schlussanträge des Generalanwalts Hogan v. 12.11.2019 - Rs. C-535/18, ECLI:EU:C:2019:957, Rdnr. 28.

140) S. zu Argumenten für die Völker- und Unionsrechtswidrigkeit der materiellen Präklusion, aber diese auch hinsichtlich Art. 9 Abs. 3 AK bejahend Guckelberger/Geber, EurUP 2014, 167, 171 ff. Dazu, dass eine Präklusionsnorm gegen Art. 9 Abs. 3 AK verstoßen „kann“, Berkemann, in: Appel/Wagner-Kardenal, Verwaltung zwischen Gestaltung, Transparenz und Kontrolle, 2019, S. 83, 139. 
dass es durchaus Stimmen gibt, die eine materielle Präklusion im Bereich des Art. 9 Abs. 3 AK für unionsrechtswidrig erachten, ${ }^{141}$ ist auch nicht völlig auszuschließen, dass die erhoffte Konsolidierung durch den EuGH in die umgekehrte Richtung ausgeht, d.h. der materiellen Präklusion im Bereich des Umweltrechtsschutzes ein Ende bereitet wird.

\subsection{Folgen für die Praxis}

In der Stellungnahme des Bundesrats vom 20.12.2019 zum Entwurf des sog. Planungsbeschleunigungsgesetzes II finden sich - anders als von den Ausschüssen angeregt - keine Regelungen zur Wiedereinführung der materiellen Präklusion. Dies ist in der Anhörung vor dem Verkehrsausschuss des Deutschen Bundestages zum Teil bedauert worden. ${ }^{142}$ Auch ist die Forderung der FDP-Fraktion in einem Antrag vom 17.12.2019 zur Wiedereinführung der bewährten Präklusionsregelung zur zeitlichen Beschränkung von Einwendungen in europarechtskonformer Weise ${ }^{143}$ ohne Erfolg geblieben. Indem der Gesetzgeber sich nicht zur Wiedereinführung der materiellen Präklusion im Umweltrecht entschieden hat, wird der Gefahr einer weiteren Unionsrechtswidrigkeit des deutschen Rechts zuvorgekommen. Die nationalen Gerichte wären bei einer solchen Neuregelung in eine missliche Lage geraten. Sie hätten die Wahl gehabt, eine derartige materielle Präklusion, weil sie von ihrer Unionsrechtswidrigkeit überzeugt sind, entsprechend der EuGH-Rechtsprechung einfach unangewendet zu lassen oder ein Vorlageverfahren an den EuGH zu starten.

Es gibt gute Gründe, zunächst die Entscheidung des EuGH in einem Vorlageverfahren der niederländischen Rechtbank Limburg an den EuGH in der Rechtssache C-826/18 abzuwarten, ob das Unionsrecht einer nationalen Regelung entgegensteht, die den Gerichtszugang gegen einen Bescheid für die betroffene Öffentlichkeit ausschließt, wenn ihr berechtigterweise der Vorwurf gemacht werden kann, keine Einwendungen gegen den Entscheidungsentwurf oder Teile davon vorgebracht zu haben. Da auch die niederländische Regelung einem Entweder-Oder-Prinzip zu folgen scheint, bleibt abzuwarten, ob sich aus dieser Entscheidung tatsächlich valide Rückschlüsse zur Beurteilung der Unionsrechtskonformität der Wiedereinführung modifizierter deutscher Präklusionsnormen ziehen lassen. ${ }^{144}$ Ein weiteres Gefahrenpotenzial eines solchen Vorhabens könnte schließlich daraus resultieren, dass neben den materiellen Präklusionsvorschriften das zur Eindämmung der Folgen der EuGH-Entscheidung in der Rs. C-137/14 erlassene Arsenal an Regelungen ( $\$ 4$ Abs. 1b, $\$ 6$ UmwRG, $\$ 7$ Abs. 5 UmwRG) weiterhin anwendbar bleibt und der Umweltrechtsschutz dadurch insgesamt zu restriktiv ausgestaltet sein könnte.

\section{Fazit}

Angesichts der Dauer von Planungsverfahren in Deutschland ist es in der Tat auch aus rechtlichen Erwägungen angebracht, über Möglichkeiten zur rascheren Realisierung von Infrastrukturvorhaben nachzudenken. Beide hier vorgestellte Überlegungen - Entscheidungen über diese per Maßnahmengesetz sowie eine mögliche Rückkehr zur materiellen Präklusion - sind unions- und völkerrechtlich äuBerst bedenklich. Gerade wegen dieser Bedenken steht zu erwarten, dass sich aufgrund solcher nur unter Involvierung des EuGH zu klärenden Fragen die jeweiligen Infrastrukturvorhaben eher langsamer realisieren lassen. Alternative Mittel zur Beschleunigung der Verfahren, wie etwa eine bessere Personalausstattung der Verwaltung ${ }^{145}$ und/oder der Gerichte, bleiben leider weiterhin ausgeblendet.

Anlässlich der anvisierten Überarbeitung der AarhusVerordnung (EG) Nr. 1367/2006 beabsichtigt die Kom- mission anscheinend, einerseits den Zugang zur administrativen und gerichtlichen Überprüfung auf Unionsebene $\mathrm{zu}$ erleichtern und andererseits Maßnahmen zur Verbesserung des Zugangs zur Justiz vor den nationalen Gerichten in allen Mitgliedstaaten zu ergreifen. ${ }^{146}$ In diesem Kontext hat der Verkehrsausschuss des Bundesrates die Bundesregierung gebeten, neben der Erfüllung der Vorgaben der AK auf die Sicherstellung zügiger und rechtssicherer Planungs- und Genehmigungsverfahren zu achten. ${ }^{147} \mathrm{Um}$ die aus Gründen des Klimaschutzes dringend erforderlichen Verkehrsinfrastrukturprojekte verwirklichen zu können, wird eine europarechtskonforme Anpassung der gerichtlichen Kontrolldichte vorgeschlagen. ${ }^{148}$ Ferner sollten Infrastrukturvorhaben, deren Notwendigkeit vom Gesetzgeber festgestellt wurde, auch realisierbar sein, wenn der Planfeststellungsbeschluss lediglich wegen eines behebbaren Verfahrensfehlers rechtswidrig ist, und die in dem MgvG angelegte Planung durch den Gesetzgeber soll selbst für weitere geeignete Sachverhalte weiterentwickelt werden, etwa durch Festlegung der Vorzugsvariante auf der Grundlage einer geeigneten Strategischen Umweltprüfung durch Bundesgesetz. ${ }^{149} \mathrm{Ob}$ derartige Ansinnen letztendlich Berücksichtigung finden, bleibt abzuwarten. Das zentrale und auch nicht abzustreitende Bedürfnis nach schnellen und zügigen Planungs- und Genehmigungsverfahren darf aber letztlich nicht dazu führen, dass immer mehr Verwaltungsentscheidungen auf den nach seiner Ausstattung und Struktur dafür weniger geeigneten Gesetzgeber verlagert werden ${ }^{150}$ und der Rechtsschutz dadurch weitestgehend ausgehöhlt wird.

Open Access. Dieser Artikel wird unter der Creative Commons Namensnennung 4.0 International Lizenz veröffentlicht, welche die Nutzung, Vervielfältigung, Bearbeitung, Verbreitung und Wiedergabe in jeglichem Medium und Format erlaubt, sofern Sie den/die ursprünglichen Autor(en) und die Quelle ordnungsgemäß nennen, einen Link zur Creative Commons Lizenz beifügen und angeben, ob Änderungen vorgenommen wurden.

Die in diesem Artikel enthaltenen Bilder und sonstiges Drittmaterial unterliegen ebenfalls der genannten Creative Commons Lizenz, sofern sich aus der Abbildungslegende nichts anderes ergibt. Sofern das betreffende Material nicht unter der genannten Creative Commons Lizenz steht und die betreffende Handlung nicht nach gesetzlichen Vorschriften erlaubt ist, ist für die oben aufgeführten Weiterverwendungen des Materials die Einwilligung des jeweiligen Rechteinhabers einzuholen.

Weitere Details zur Lizenz entnehmen Sie bitte der Lizenzinformation auf http://creativecommons.org/licenses/by/4.0/deed.de.

Open Access funding enabled and organized by Projekt DEAL.

141) S. auch Schlussanträge des Generalanwalts Hogan v. 12.11.2019 - Rs. C-535/18, ECLI:EU:C:2019:957, Rdnr. 28.

142) Stellungnahme der IHK Nord für den Deutschen Bundestag, Ausschuss für Verkehr und digitale Infrastruktur, Ausschussdrucks. 19(15)308-C; Stellungnahme von Bergmann/DB Netz AG für den Deutschen Bundestag, Ausschuss für Verkehr und digitale Infrastruktur, Ausschussdrucks. 19(15)308-H.

143) BT-Drs. 19/16040, S. 2.

144) Durner, Rechtsgutachten zur Wiedereinführung der materiellen Präklusion v. 7.10.2019, S. 29.

145) Zu Letzterem Wysk, in: Appel/Wagner-Kardenal, Verwaltung zwischen Gestaltung, Transparenz und Kontrolle, 2019, S. $47 \mathrm{ff}$.

146) So Empfehlungen BR-Drs. 655/1/19, S. 29.

147) Empfehlungen BR-Drs. 655/1/19, S. 28.

148) Empfehlungen BR-Drs. 655/1/19, S. 28

149) Empfehlungen BR-Drs. 655/1/19, S. 29.

150) $\mathrm{Zu}$ dieser stärker werdenden Tendenz auch Baumann/Brigola, DVB1. 2020, 324, 326. 